\title{
A model for the fluid dynamic behavior of a film coating suspension during tablet coating
}

\author{
C. Christodoulou ${ }^{a}$, E. Sorensen ${ }^{a}$, A. S. Khair ${ }^{c}$, S. García-Muñoz $^{b}$, L. Mazzei $^{a \dagger}$ \\ ${ }^{a}$ Department of Chemical Engineering, University College London, Torrington Place, London WC1E 7JE, UK \\ ${ }^{b}$ Eli Lilly and Company, Lilly Research Laboratories, Indianapolis, IN 46285, USA \\ ${ }^{c}$ Department of Chemical Engineering, Carnegie Mellon University, Pittsburgh, PA 15213, USA
}

\begin{abstract}
This work models the behavior of a liquid-particle suspension on the surface of a tablet during the pharmaceutical film-coating process. The model uses the "mixture modeling" approach and the lubrication approximation method to simulate how the suspension moves and dries on the surface of a tablet, considering how important physical properties of the suspension, such as the density and viscosity, change when the carrier fluid evaporates. The model also accounts for the absorption of the coating suspension inside the core of the porous tablet, yielding the tablet water content, a key quantity characterizing the coating process. The numerical results, obtained with the gPROMS Modelbuilder platform, agree with experimental data taken from the literature and Volume-Of-Fluid CFD simulations.
\end{abstract}

\section{Introduction}

Coating of tablet cores is one of the oldest manufacturing processes used by the pharmaceutical industry (Cole et al., 1995). Among the various coating methods, aqueous film-coating has recently gained in popularity (Felton, 2013). During this process, a suspension of water and polymer particles is atomized and sprayed on tablets lying in a rotating drum. Following spray impact, the droplets of coating suspension spread and form thin films onto the surfaces of the tablets that are facing the spray (tablet wetting). After the initial wetting, the continuous phase of the suspension (water) evaporates, while the suspension absorbs in the porous tablets (Felton, 2013). Once water has sufficiently depleted from the tablet surfaces, a dry polymer film generates (Muliadi and Sojka, 2010). As the tablets move inside the rotating drum, they pass under the spray several times, each time with different orientation. Owing to the this variability in tablet orientation, the surfaces facing the spray frequently change, so in the end the tablets are entirely coated. The process completes when the dry polymer films reach the desired thickness $\left(\sim 10^{-5}\right.$ to $10^{-4} \mathrm{~m}$; Freireich et al., 2015).

Owing to the complexity of the coating process, the final product often presents defects, the most common being coating bridging, cracking, orange-peel roughness and picking/sticking (Cole et al., 1995). These defects arise primarily because the values of process parameters, such as spray flow rate, drum rotation speed, temperature and relative humidity, are chosen incorrectly; this leads to an uneven distribution of the coating on the tablet surface (especially at the tablet edges) and so to final product imperfections. In particular, when the water drying rate is uncontrolled, overwetting and overdrying problems are likely to occur (especially close to the tablet edges) and the coating process may take longer (Muliadi and Sojka, 2010).

To investigate the behavior of a coating suspension on a pharmaceutical tablet, one needs to consider its flow over the surface and edges of the tablet, its evaporation from the surface of the tablet, and its absorption into the porous matrix of the tablet. Previous work concerning the simulation of thin films over rigid substrates either dealt with pure liquids or neglected the absorption of the suspension and/or the evaporation of its continuous phase (i.e. the carrier fluid). In this work, we aim to account for all these concurrent phenomena, because all of them strongly affect the tablet coating process.

\footnotetext{
${ }^{\dagger}$ Corresponding author. Tel. +44(0)207679 4328; Email: l.mazzei@ucl.ac.uk
} 
The wetting and spreading phenomena of pure liquid films on impermeable, non-porous substrates have been investigated employing the lubrication approximation method (de Gennes, 1985; Schwartz, 1999; Craster and Matar, 2009). This method exploits the small value of the ratio between the thickness and the width of a film to derive a set of simplified continuity and Navier-Stokes equations describing the film flow in the absence of inertia. Alleborn and Raszillier (2004) extended this method to investigate how droplets spread over porous substrates while absorbing into them.

Many other researchers have developed mathematical models for the absorption of pure liquids into porous substrates. Most of their work is based on the Lucas - Washburn or Darcy's equations, which allow predicting the spreading and penetration depth of the liquid in the porous medium (Siregar, 2012). In our previous work (Christodoulou et al., 2018), we developed a model that yields the wetting front profile inside a porous pharmaceutical tablet after a pure water droplet impinges on its surface.

However, most coating formulations employed by the pharmaceutical industry are not pure liquids, but aqueous polymer suspensions. To model their flow on the surface of pharmaceutical tablets, one can adopt the "mixture modeling" approach. This holds for fluid-particle systems in which the mean velocity fields of the continuous and disperse phases relax to local dynamical equilibrium rapidly, a condition that is often met in liquid-particle suspensions (Jamshidi et al., 2019). Weidner et al. (1996) and O'Brien and Schwartz (2002) combined this approach and the lubrication approximation method to simulate the drying of suspension films and their flow over impermeable substrates.

Absorption models for suspensions in porous media should take into account particle retention (Bradford et al., 2002). This phenomenon has been investigated theoretically and experimentally by many researchers, such as Bradford et al. (2003), Civan (2011) and Holloway et al. (2011). Even though their work is not specific to pharmaceutical formulations, it provides a valuable theoretical background for the modeling of their absorption in pharmaceutical tablets.

The main limitation of the models described above is that they neglect evaporation, a phenomenon that is crucial at the high temperature at which the film-coating process takes place and that generates a dry crust over the tablet surface (Felton, 2013). Both phenomena are well described by the models of Croll (1987) and Kiil (2006). More recently, Niblett et al. (2017) studied the application and drying of coating suspensions on tablets both experimentally and numerically, presenting a model that predicts the suspension drying rate. Their model, among other things, simulates droplet application and drying on the tablet, and their numerical results are validated against data from the actual process.

Recent models which simulate coating application on tablets have focused on single droplet impact cases and/or have neglected coating evaporation and absorption into the tablet (Bolleddula et al., 2010; Niblett et al., 2017). In this work, we developed a model that is able to predict the behavior of a pharmaceutical suspension film after it is applied on a tablet during a pass under the spray, accounting for film spreading, suspension absorption with particle retention and carrier fluid evaporation. All these phenomena should be considered to accurately predict the amount of water and solid polymer inside the tablet core. This model can be used by the pharmaceutical industry (together with models describing tablet movement under the spray, as well as spray atomization and evaporation) to understand the process better and enhance tablet shelf-life and dry film adhesion.
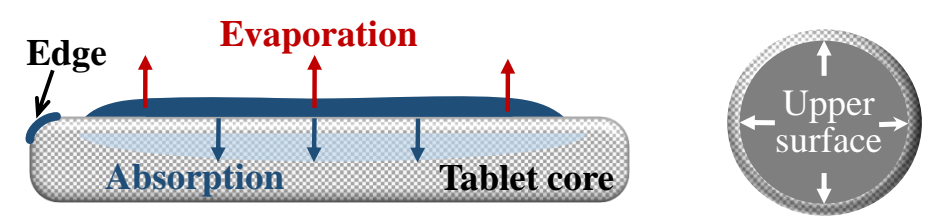

Figure 1: Coating film applied on the upper surface of a cylindrical tablet. 


\section{Mathematical model}

Film coatings are generally applied on pharmaceutical tablets by spraying a polymer-based suspension on their surface. The atomized suspension may dry significantly during the flight from the spray nozzle to the tablet passing under the spray; in some cases, the drying can be substantial to the point that some droplets are effectively "spray-dried". After impinging onto the tablet, the liquid droplets spread on its dry surface (Christodoulou et al., 2020). As droplets continuously impinge, the wetted area on the tablet expands, eventually forming a liquid (suspension) film that covers part of the tablet surface facing the spray (upper surface in Fig. 1). According to the work of Bolleddula et al. (2010), this process is driven by inertia and its time scale (milliseconds) is far shorter than those (seconds) of the absorption and drying processes (Felton, 2013).

Following the inertia-driven spreading, the coating suspension penetrates into the core of a porous tablet and flows over adjacent dry parts of the tablet surface; concurrently, the carrier fluid evaporates from the film surface. This last phenomenon strongly affects the motion of the film (absorption into the tablet pores and spreading over the dry parts of the tablet surface), because it raises the solid volume fraction in the suspension, and with it the suspension density and viscosity. The viscosity, in particular, diverges when the solid volume fraction increases sufficiently (Mueller et al., 2009). The critical value at which this happens has not been precisely identified; following Rutgers (1962a), here we take it to be 0.67 , which is in the range of accepted values. When the solid volume fraction reaches this value, the particles come into close contact, start coalescing and form a porous wetted solid (Taylor and Winnik, 2004). At this point, the carrier fluid evaporates through the pores of the wetted solid or "crust", while the film essentially stops absorbing into the tablet and flowing over its surface (Kiil, 2006). After the film has dried, the tablet passes again under the spray (probably with different orientation) and the above process repeats itself until the dry coating film reaches the desired thickness on all the surfaces of the tablet.

In this article, we are interested in the behavior of a pharmaceutical coating suspension (consisting of water and particles) after this has been applied on a tablet that is no longer under a spray; that is, after the initial inertia-driven spreading process has completed. The inertia-driven regime was studied in our previous work (Christodoulou et al., 2020). We assumed that the tablet is cylindrical (even though the model can work for tablets of different shapes) and initially the suspension film covers part of its flat "upper" surface and of its edges (Fig. 1). The film can then penetrate into the tablet and flow over its flat surface and curved edges, while the suspension carrier fluid can evaporate. The model accounts for the particle retention in the pores, since this may hinder the suspension from absorbing into the core. We regarded a simulation as completed when the particle concentration everywhere in the coating film reaches its critical value and the suspension turns into a porous wetted crust (Kiil, 2016). After the crust has formed, water evaporates from it; this process is not investigated because it was studied in our previous work (Christodoulou et al., 2018).

To summarize, the model presented here can calculate the time required by the coating film to turn into a crust, the amount of coating suspension absorbed into the tablet core, the thickness of the film as it evaporates, flows over the tablet and penetrates into its pores, and the profile of the solid volume fraction within the film and inside the tablet. All the above predictions concern a single pass of the tablet under the spray. In this section we describe the mathematical model; this is presented in three subsections: Subsections 2.1 and 2.2 concern the behavior of the coating film on the flat surface and on the edges of the tablet, respectively, and Subsection 2.3 deals with the penetration of the coating suspension into the porous tablet. Subsection 2.4 reports the steps needed to solve the model numerically. 


\subsection{Coating behavior on a flat tablet surface}

After a coating suspension film has been applied on a flat region of the tablet surface, it can spread over the adjacent flat regions of the surface or over the adjacent edges (see Subsection 2.2), absorb into the porous tablet core, and dry while the coating carrier fluid (water) evaporates (Fig. 1). In this subsection, we present the part of our mathematical model that describes how the suspension flows and dries over the adjacent flat regions of the tablet surface.

Pharmaceutical coatings are multiphase media (liquid-particle suspensions). To simplify the problem, we assumed that the coating film consists of one carrier liquid (liquid phase) containing identical particles (solid phase). We also assumed that the velocity fields of the two phases relax rapidly; this is true in the simulations performed in this study, because the Stokes number is small (St $<<1$; see Appendix). The last assumption allowed us to use the mixture modeling approach. This approach is based on a set of four balance equations, which are reduced to three by expressing the slip velocity between the phases with a constitutive equation. The remaining balance equations are two continuity equations (one for the mixture, treated as an effective fluid, and one for the solid phase) and one linear momentum balance equation for the mixture.

To obtain the continuity equation for the mixture one has to sum the continuity equations written for the solid and liquid phases (Jamshidi et al., 2019). Doing so yields:

$$
\partial_{t} \rho=-\partial_{\boldsymbol{x}} \cdot(\rho \boldsymbol{u})
$$

where $\rho$ and $\boldsymbol{u}$ are the density and velocity of the mixture (that is, of the coating suspension), respectively, and are defined as follows:

$$
\rho \equiv \varepsilon \rho_{e}+\phi \rho_{s} ; \rho \boldsymbol{u} \equiv \varepsilon \rho_{e} \boldsymbol{u}_{e}+\phi \rho_{s} \boldsymbol{u}_{s}
$$

where $\varepsilon$ and $\rho_{e}$ are the volume fraction and density of the liquid phase, respectively, and $\phi$ and $\rho_{s}$ are the volume fraction and density of the solid phase, respectively. Moreover, $\boldsymbol{u}_{e}$ and $\boldsymbol{u}_{s}$ are the velocities of the liquid and solid phases, respectively.

To derive a linear momentum balance equation for the mixture one needs to follow the same procedure as for the continuity equation. Summing the dynamical equations for the fluid and solid phases yields the equation of motion for the coating suspension:

$$
\partial_{t}(\rho \boldsymbol{u})=-\partial_{\boldsymbol{x}} \cdot(\rho \boldsymbol{u u})+\partial_{\boldsymbol{x}} \cdot \boldsymbol{\sigma}_{m}+\rho \boldsymbol{g}
$$

where $\boldsymbol{g}$ is the gravitational field and $\boldsymbol{\sigma}_{m}$ is the mixture stress tensor. The equation above is unclosed; to make it solvable, one needs a constitutive equation for the mixture stress tensor. We discuss this equation below, in this subsection.

As mentioned before, an additional continuity equation for the solid phase (particles in the suspension) is needed to model the behavior of the coating film. Since the coating particles are incompressible, this equation can be written as follows:

$$
\partial_{t} \phi=-\partial_{\boldsymbol{x}} \cdot(\phi \boldsymbol{u})-\partial_{\boldsymbol{x}} \cdot \boldsymbol{j}
$$

The term $\partial_{\boldsymbol{x}} \cdot \boldsymbol{j}$ arises because the particles do not move at the same velocity as the mixture. $\boldsymbol{j}$ can be interpreted as a particle migration flux and its closure is also discussed below. 


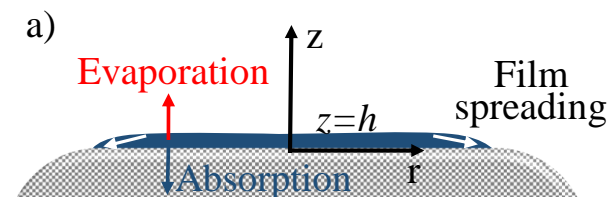

b)

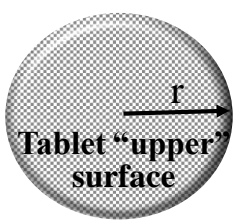

Figure 2: a) Coating film covering part of the "upper" tablet surface facing the spray,

b) Tablet "upper" surface during a single pass under the spray.

By solving Eqs. (1), (3) and (4) along with appropriate expressions for $\boldsymbol{\sigma}_{m}$ and $\boldsymbol{j}$, one can determine the solid volume fraction and velocity of the mixture. But solving these is computationally demanding. To reduce the computational effort without sacrificing accuracy, one can employ the lubrication approximation method, a technique that exploits the small value of the ratio between the thickness and the width of a film to simplify its equations of motion. In this method, the modeler identifies, via scaling and order-of-magnitude analysis, and then neglects terms that are too small to affect the dynamics of the suspension. For the present problem, these terms turn out to be inertia and gravity (Szeri, 2010).

In the literature it is reported that pharmaceutical suspension films formed by impinging droplets are approximately cylindrical (Niblett et al., 2017). Assuming this shape for the film and employing the lubrication approximation method, we obtained the following dynamical equations, which we express in cylindrical coordinates:

$$
\partial_{r} p=\partial_{z} \tau_{z r} \quad ; \quad \partial_{z} p=0
$$

where $r$ and $z$ are the radial and axial (vertical) coordinates, respectively (Fig. 2), $p$ is the pressure of the mixture and $\tau_{z r}$ is the $z r$-component of the deviatoric stress tensor of the mixture. Eqs. (5a) and (5b) are derived in the appendix. The second indicates that the pressure does not depend on the $z$ coordinate; therefore, assuming axisymmetry, we conclude that $p$ is a function of the radial coordinate and of the time only.

In the literature, pharmaceutical coating suspensions have been reported to exhibit either Newtonian or slight shear-thinning behavior (Bolleddula et al., 2010; Ketterhagen et al., 2017; Niblett et al., $2017)$ over a range of shear rates $\left(10^{-3}-10^{3} 1 / \mathrm{s}\right)$ of interest for the coating-application process. The rheology of these suspensions depends on their composition (type of polymer) and solid volume fraction (Cole et al., 1995). In this work, we considered power-law suspensions, because these can capture both Newtonian and shear-thinning behavior. We thus write:

$$
\tau_{z r}=m\left|\partial_{z} v\right|^{n-1} \partial_{z} v
$$

where $v$ is the radial component of the mixture velocity, $m$ is the consistency index and $n$ is the flow index. Substituting Eq. (6) into Eq. (5a) gives:

$$
\partial_{r} p=\partial_{z}\left[m\left|\partial_{z} v\right|^{n-1} \partial_{z} v\right]
$$

We now integrate Eq. (7) twice between $z=0$ (the tablet-film interface), where we impose no-slip, and $z=h$ (the film-air interface), where we impose no-shear-stress. With the boundary conditions just specified, which read:

$$
\left.v\right|_{z=0}=0 \quad ;\left.\quad \partial_{z} v\right|_{z=h}=0
$$

we obtain:

$$
v=\frac{n}{n+1}\left(-\frac{\partial_{r} p}{m}\right)^{1 / n}\left[h^{(n+1) / n}-(h-z)^{(n+1) / n}\right]
$$

Setting $n=1$ gives the expression for a Newtonian fluid. In Eq. (9), the pressure field is unknown; 
to determine it, we use the following equation that accounts for the pressure jump at the film-air interface due to surface tension:

$$
p=-\gamma \partial_{\boldsymbol{x}} \cdot \partial_{\boldsymbol{x}} h
$$

where $\gamma$ is the surface tension at the film-air interface (assumed to be constant). Eq. (10) was employed by O'Brien and Schwartz (2002), who investigated the flow of films over impermeable substrates. When the substrate is flat, the curvature of the film-air interface is approximately equal to $\partial_{\boldsymbol{x}} \cdot \partial_{\boldsymbol{x}} h(r, t)$, which in cylindrical coordinates yields $(1 / r) \partial_{r}\left(r \partial_{r} h\right)$.

As the film flows over the tablet surface, it spreads over a dry substrate. This "contact-line" motion does not agree with the no-slip boundary condition at the film-tablet interface, which we employed to derive the velocity profile in Eq. (9). To overcome this problem, we followed the approach of Schwartz and Eley (1998), who assumed that a precursor film of coating suspension covers the dry substrate. Inside the submicroscopic precursor film, a "disjoining" pressure $\pi_{c}$ arises (Slattery et al., 2007); this accounts for the Van der Waals forces between the film and the tablet and is significant at the contact line. We calculated the disjoining pressure using the Frumkin-Derjaguin model (Schwartz, 1999):

$$
\pi_{c}=\beta\left[\left(\frac{\hat{h}}{h}\right)^{N}-\left(\frac{\hat{h}}{h}\right)^{M}\right] \quad ; \quad \beta=\frac{1}{\hat{h}} \frac{(N-1)(M-1)}{2(N-M)} \gamma(1-\cos \vartheta)
$$

where $\hat{h}$ is the precursor film thickness, $N$ and $M$ are positive constants with $N>M>1$ and $\vartheta$ is the equilibrium contact angle of the film on the tablet. Following Schwartz et al. (2001), we took $N$ and $M$ equal to 3 and 2, respectively. The precursor film thickness $\hat{h}$ is much smaller than the coating film thickness $(\hat{h}=0.1 \mu \mathrm{m})$. Modeling the disjoining pressure using the Frumkin-Derjaguin model (Eq. 11) allowed us to prescribe the equilibrium contact angle. The final pressure equation reads:

$$
p=-\gamma \partial_{\boldsymbol{x}} \cdot \partial_{\boldsymbol{x}} h-\pi_{c}
$$

Here, the thickness of the film is unknown but can be obtained from the mass balance equation for the mixture, Eq. (1). If we use a scaling analysis, this can be approximated as follows:

$$
\partial_{t} \rho=-(1 / r) \partial_{r}(r \rho v)-\partial_{z}(\rho w)
$$

where $w$ is the axial component of the mixture velocity. Integrating Eq. (13) with respect to $z$, from $z=0$ to $z=h$, gives:

$$
\partial_{t}(\rho h)=-(1 / r) \partial_{r}(\rho r Q)-\rho W_{a}-\dot{m}_{e}
$$

The derivation of Eq. (14) and the necessary boundary conditions are given in the Appendix; in this equation, $W_{a}$ is the absorption velocity, $\dot{m}_{e}$ is the evaporation mass flux and:

$$
Q \equiv \int_{0}^{h} v d z
$$

To solve Eq. (14), we need to assign an initial condition and two boundary conditions. The former poses no problem, if the initial shape of the film is known; we assumed that the film initially has a cylindrical shape (Niblett et al., 2017). The latter are easily specified at $r=0$, where the gradient of $h$ vanishes by symmetry, and at the edge of the film, where the thickness of the film is equal to the precursor film thickness $h=\hat{h}$. Film spreading over the curved edges of a tablet cannot be described by Eq. (14). This is because the cylindrical coordinate system does not hold for the flow over the edges. For that reason, we derived another evolution equation that can calculate $h$ at the end of the flat "upper" surface and over the tablet edges (see Subsection 2.2). 
Finally, to solve Eq. (14), one needs expressions for the absorption velocity and the evaporation mass flux. We calculated the former from the boundary condition at the film-tablet interface:

$$
W_{a}=\varphi w_{p} \text { at } z=0
$$

where $w_{p}$ is the (volume averaged) mixture velocity in the vertical direction inside the tablet (discussed in Subsection 2.3) and $\varphi$ is the porosity of the tablet.

To calculate the evaporation mass flux $\dot{m}_{e}$, we assumed that as the film dries, and before the wetted crust forms, the carrier fluid finds its way to the film-air interface without being hindered by the particles (Kiil, 2006). The evaporation flux from a thin film is approximately constant over the film-air interface (Weidner et al. 1996; Kiil, 2006) and given by:

$$
\dot{m}_{e}=\frac{k_{m} \mathcal{M}_{w}}{\Re}\left[\frac{p_{\nu}^{*}\left(T_{s}\right)}{T_{s}}-\frac{R H p_{\nu}^{*}\left(T_{\infty}\right)}{T_{\infty}}\right]
$$

where $k_{m}$ is the mass transfer coefficient, $\mathcal{M}_{w}$ is the molecular weight of the evaporating liquid phase (water), $R H$ is the relative humidity in the air bulk, $\Re$ is the universal gas constant, and $p_{\nu}^{*}$ is the saturated vapor pressure calculated at the film-air interface temperature $T_{s}$ and at the bulk gas temperature $T_{\infty}$. Eq. (17) neglects the film curvature effect on the evaporation rate (negligible Kelvin effect), an approximation already adopted in the literature for similar systems (Weidner et al., 1996; O'Brien and Schwartz, 2002). The mass transfer coefficient $k_{m}$ can be estimated with the Ranz-Marshall correlation (Kiil, 2006):

$$
\mathrm{Sh}=2+0.65 \operatorname{Re}^{1 / 2} \mathrm{Sc}^{1 / 3} ; \text { for } \operatorname{Re}<3 \cdot 10^{5}
$$

where Sh, Re and Sc are the Sherwood, Reynolds and Schmidt numbers, respectively. In Eq. (18), which is valid for laminar flow of thin films (Kiil, 2006), the Reynolds and Schmidt numbers are defined as follows:

$$
\operatorname{Re} \equiv \frac{\rho_{g} v_{g} L_{s}}{\mu_{g}} ; \quad \mathrm{Sc} \equiv \frac{\mu_{g}}{\rho_{g} \mathcal{D}_{\nu}}
$$

where $\rho_{g}, \mu_{g}$ and $v_{g}$ are the density, viscosity and characteristic velocity of the gas (air + vapor) above the tablet, respectively, $\mathcal{D}_{\nu}$ is the vapor diffusivity and $L_{s}$ is the characteristic length of the film. In our simulations, $L_{s}$ was taken to be equal to the diameter of the wetted area of the coating film covering the surface of the tablet. We obtained the vapor diffusivity $\mathcal{D}_{\nu}\left(\mathrm{m}^{2} / \mathrm{s}\right)$ using the following empirical relation (Mezhericher et al. 2007):

$$
\mathcal{D}_{\nu}=3.564 \cdot 10^{-10}\left(T_{s}+T_{\infty}\right)^{1.75}
$$

To calculate the evaporation flux $\dot{m}_{e}$ (Eq. 17), one needs to estimate the temperature at the film surface $T_{s}$. Following Kiil (2006), we assumed that the temperature $\left(T_{1}\right)$ is uniform throughout the film, since this is very thin. We then calculated $T_{s}=T_{1}$ from the following energy balance equation:

$$
\left\langle\rho c_{p 1}\right\rangle V_{1} \partial_{t} T_{1}=-\dot{m}_{e} \Delta H_{e} A_{12}-h_{H}\left(T_{1}-T_{2 \infty}\right) A_{12}-\left.k_{3} \partial_{z} T_{3}\right|_{z=0} A_{13}
$$

where $A_{12}$ and $A_{13}$ are the areas of the film-air and film-tablet interfaces, respectively, $V_{1}$ is the volume of the film, $c_{p 1}$ is the heat capacity of the film, $T_{3}$ is the temperature field within the tablet, $h_{H}$ is the heat transfer coefficient, $k_{3}$ is the thermal conductivity of the tablet and $\Delta H_{e}$ is the enthalpy of vaporization. Eq. (21) refers to a control volume coinciding with the entire film. The equation is derived in the appendix, where the heat conduction term (the last on the right-hand side) is also analyzed. 
The solid volume fraction field in the film indicates how uniform this is. An evolution equation for this field is obtained from Eq. (4), the continuity equation for the solid phase of the mixture. In cylindrical coordinates, this reads:

$$
\partial_{t} \phi=-(1 / r) \partial_{r}(r \phi v)-\partial_{z}(\phi w)-(1 / r) \partial_{r}\left(r j_{r}\right)-\partial_{z} j_{z}
$$

where $j_{r}$ and $j_{z}$ denote the components of the particle migration flux in the radial and axial directions, respectively. Particle migration in the axial direction can be neglected, because in this direction the solid volume fraction varies mildly (Weidner et al., 1996). Eq. (22) then reduces to:

$$
\partial_{t} \phi=-(1 / r) \partial_{r}(r \phi v)-\phi \partial_{z} w-(1 / r) \partial_{r}\left(r j_{r}\right)
$$

Similar steps to those presented in the derivation of Eq. (14) (see Appendix) yield the following equation:

$$
\partial_{t} \phi=-\frac{\rho Q}{h} \partial_{r}(\phi / \rho)+\frac{\phi \dot{m}_{e}}{\rho h}-\frac{1}{h} \frac{1}{r} \partial_{r}\left(r h j_{r}\right)+\frac{\phi}{\rho} \partial_{t} \rho
$$

To solve Eq. (24), we need a constitutive equation for the flux $j_{r}$. To derive it, we first need to estimate the particle Peclet number $\mathrm{Pe} \equiv a^{2} \dot{\gamma} / \mathcal{D}$, where $\mathcal{D}$ is the particle Brownian diffusion coefficient, $a$ is the particle radius (assuming the particles are spherical) and $\dot{\gamma}$ is the scale of the shear rate (that is, of the magnitude of the rate of deformation tensor). For the cases considered in this work, $\mathrm{Pe} \sim 10^{-6}$ (see Appendix). Because Pe is much less than unity, the coating suspension is Brownian and the following constitutive equation applies:

$$
j_{r}=-\mathcal{D} \partial_{r} \phi
$$

where $\mathcal{D}$ is a function of the solid volume fraction (Batchelor, 1975; Yiantsios et al., 2006). Following Buyevich and Kapbsov (1999), we used the following expression to calculate $\mathcal{D}$ :

$$
\mathcal{D}=\mathcal{D}_{0} / \chi(\phi) \quad ; \quad \chi(\phi)=\frac{1-0.5 \phi}{(1-\phi)^{3}}+1.08 \frac{\left(\phi / \phi^{*}\right)^{3}}{1-\phi / \phi^{*}}
$$

where $\phi^{*}$ represents the solid volume fraction at maximum packing, equal to 0.67 for hard spheres, and $\mathcal{D}_{0}=\left(k_{B} T_{w}\right) /(6 \pi \mu a)$ is the Einstein diffusivity, where $k_{B}$ is the Boltzmann coefficient, $T_{w}$ and $\mu_{w}$ are the carrier fluid temperature and viscosity, respectively. When the value of $\phi$ reaches $\phi^{*}$, the diffusion coefficient approaches zero and no particle migration takes place (Buyevich and Kapsov, 1999). According to Buyevich and Kapsov, Eq. (26) holds for the whole region of $\phi$ considered in this article $\left(0.1 \rightarrow \phi^{*}\right)$ and thus offers a good estimate for the concentration-dependent Brownian diffusivity.

As mentioned before, pharmaceutical coatings can be Newtonian or slightly shear-thinning (Bolleddula et al., 2010; Ketterhagen et al., 2017). In the latter case, since the mixture viscosity depends on the solid volume fraction, we must write:

$$
\eta(\dot{\gamma}, \phi)=m(\phi)\left|\partial_{z} v\right|^{n(\phi)-1}
$$

where $\eta$ is the non-Newtonian mixture viscosity, while $m(\phi)$ and $n(\phi)$ are rheometric functions that must be determined experimentally (Mueller et al., 2010).

For coating formulations that behave as Newtonian fluids, the mixture viscosity is a function of the solid volume fraction only. A constitutive equation that allows calculating it is that of Krieger and Dougherty (1959), which reads:

$$
\mu(\phi)=\mu_{w}\left(1-\phi / \phi^{*}\right)^{-1.82}
$$

where $\mu$ is the Newtonian mixture viscosity. 
Eq. (28) is accurate for Brownian suspensions with solid volume fractions up to 0.55; for denser suspensions, the equation of Brady (1993) applies:

$$
\mu(\phi)=1.3 \mu_{w}\left(1-\phi / \phi^{*}\right)^{-2}
$$

When the film temperature increases, the mixture viscosity decreases (Ketterhagen et al., 2017). This effect is captured by the following constitutive equation:

$$
\eta\left(\dot{\gamma}, \phi, T_{1}\right)=\left.\eta(\dot{\gamma}, \phi)\right|_{20^{\circ} \mathrm{C}} \cdot \exp \left[A_{c} /\left(T_{1}-T_{c}\right)\right]
$$

where $A_{c}\left({ }^{\circ} \mathrm{C}\right)$ and $T_{c}\left({ }^{\circ} \mathrm{C}\right)$ are coefficients determined experimentally (Civan, 2008b), and $\left.\eta(\dot{\gamma}, \phi)\right|_{20^{\circ} \mathrm{C}}$ is given by Eq. (27), or Eqs. (28) and (29) for the Newtonian case.

The simulation of the film flow was stopped when the suspension reached maximum packing. At this point, the suspension film can no longer be regarded as a liquid (the viscosity becomes infinite) but as a wetted solid - at this stage, a wetted crust forms in the entire thin film.

In this subsection, we presented the part of our model describing how the suspension flows and dries. Eqs. (14) and (24) yield the film thickness and the solid volume fraction profiles in the film over the surface of the tablet. However, they can be solved only if the absorption velocity $\left(W_{a}\right)$ at the film-tablet boundary is known (see Eq. 16). In Subsection 2.3, we investigate the absorption of the coating suspension into the tablet and derive an expression for $W_{a}$.

\subsection{Coating behavior on a tablet edge}

When tablets are sprayed, their flat regions are exposed more than their edges (Freireich et al., 2015); the latter, therefore, are less covered with coating suspension and may even remain dry. The flow over these peripheral regions is driven by the large gradients in surface curvature present over the edges of the tablet (Roy et al., 2002). In the case of a film covering a convex tablet edge, the curvature gradient is expected to drive the suspension away from the edge thus leading to film thinning (Schwartz and Weidner, 1995) and to tablet defects (Freireich et al., 2015). In this subsection we investigate this flow, considering edges that initially are either dry or lightly coated.

To describe the flow over the edges of the tablet, we introduced an orthogonal curvilinear coordinate system $\left(s_{1}, s_{2}, z\right)$, where the coordinate directions are chosen as shown in Fig. 3. For most tablet shapes (cylindrical, round biconvex, etc.), the surface curvature at the edge in the $s_{1}$ direction is much larger than that in the $s_{2}$ direction. We can thus neglect the flow in the $s_{2}$ direction and treat the problem as a two-dimensional flow in the $\left(s_{1}, z\right)$ plane, as shown in Fig. 4.

Following Schwartz and Weidner (1995), who simulated thin film flow over curved surfaces using the curvilinear two-dimensional coordinate system described above, we write the evolution equation for the film thickness $h_{c}$ over the tablet edge as follows:

$$
\partial_{t}\left(\rho h_{c}\right)=-\partial_{s_{1}}\left(\rho Q_{c}\right)-\rho W_{a}-\dot{m}_{e}
$$

where the absorption velocity $W_{a}$ and the water evaporation flux $\dot{m}_{e}$ are given by Eqs. (16) and (17), respectively, and:

$$
Q_{c} \equiv \int_{0}^{h} v_{c} d z
$$

where the velocity component $v_{c}\left(s_{1}, z, t\right)$ is given by the equivalent of Eq. (9) written in the coordinate system of the edge: 

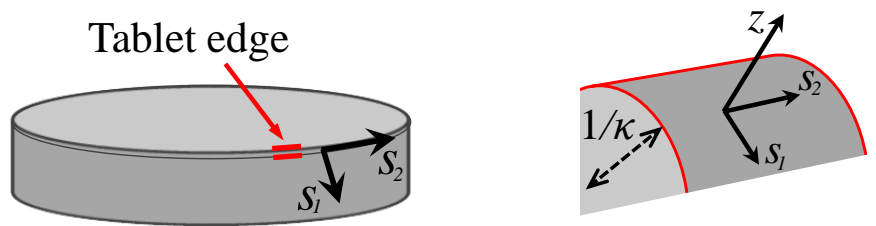

Figure 3: Curvilinear coordinate system for the tablet edge.

$$
v_{c}=\frac{n}{n+1}\left(-\frac{\partial_{s_{1}} p}{m}\right)^{1 / n}\left[h_{c}^{(n+1 / n)}-\left(h_{c}-z\right)^{(n+1 / n)}\right]
$$

In Eq. (33) the pressure field is unknown. Following Roy et al. (2002) and accounting for the disjoining pressure, we can calculate the pressure in the film over the edge using the following equation:

$$
p=-\gamma\left[\kappa+\kappa^{2} h_{c}+\partial_{s_{1}}^{2} h_{c}\right]-\pi_{c}
$$

where $\kappa$ is the curvature of the $2 \mathrm{D}$ edge (Fig. 3).

At the point where the flat surface and the tablet edge meet $\left(s_{1}=0\right)$ the curvature $\kappa$ is zero. We considered that at this point it is $h_{c}=h\left(r=R_{t}\right)$, where $h$ is the thickness of the film covering the flat surface of the tablet and $R_{t}$ is the radius of the flat tablet surface (Subsection 2.1). Additionally, similarly to the case of coating flow on a flat surface, we assumed that at the edge of the film, $h_{c}$ is equal to the precursor film thickness $\hat{h}$.

The evolution equation for the solid volume fraction inside a suspension flowing over a $2 \mathrm{D}$ edge is given by Schwartz and Weidner (1995):

$$
\partial_{t} \phi=-\frac{\rho Q_{c}}{h_{c}} \partial_{s_{1}}(\phi / \rho)+\frac{\phi \dot{m}_{e}}{\rho h_{c}}-\frac{1}{h_{c}} \partial_{s_{1}}\left(h_{c} j_{s_{1}}\right)+\frac{\phi}{\rho} \partial_{t} \rho
$$

where the component of the particle migration in the $s_{1}$-coordinate is given by:

$$
j_{s_{1}}=-\mathcal{D} \partial_{s_{1}} \phi
$$

In this subsection, we presented the part of our model which deals with the suspension flow and drying over a curved edge of the tablet. With Eqs. (31) and (35), one can predict the film thickness and the particle volume fraction profiles over the edge of the tablet.

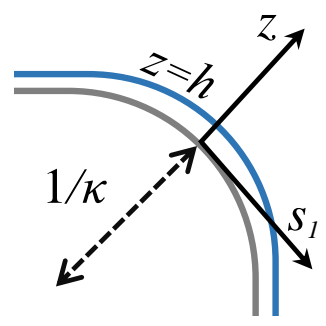

Figure 4: Flow over the tablet edge.

\subsection{Coating behavior into the tablet core}

As mentioned before, the coating suspension penetrates into the porous tablet at the same time as it dries and flows over its surface. Since the characteristic size of the particles of a coating suspension ( $a \sim 10^{-8}$ to $10^{-9} \mathrm{~m}$; Cole et al., 1995) is much smaller than the diameter of the tablet pores $\left(d_{p} \sim 10^{-6} \mathrm{~m}\right.$; Collins et al., 2007), the pores do not clog immediately. However, particle retention on the pore walls takes place and reduces the permeability of the tablet. When the coating suspension dries at the surface of the tablet, the imbibition stops. 
In this subsection, we present the part of the model which predicts the wetting front depth inside the tablet. Here, we neglected coating absorption from the tablet edges, because the amount of suspension that penetrates into the tablet from the edges is negligible compared to the overall amount of suspension absorbed, a consequence of the wetted area of the flat tablet surface being much larger than that of the tablet edges. To calculate the wetting front depth $\left(h_{p}\right)$, we need expressions for the suspension velocity $\left(\boldsymbol{u}_{p}\right)$ and pressure $\left(p_{p}\right)$ fields inside the tablet. Additionally, we must account for the effect of particle retention on the porosity and permeability of the porous tablet. For that reason, it is necessary to calculate the volume fraction of the particles in the suspension $\left(\phi_{p}\right)$ and of the particles deposited on the walls of the pores $\left(\epsilon_{p}\right)$.

To calculate $\boldsymbol{u}_{p}$, we used Darcy's equation:

$$
\boldsymbol{u}_{p}=-\frac{1}{\varphi} \frac{\mathcal{K}_{p}}{\eta} \partial_{x} p_{p}
$$

where $\varphi$ is the tablet porosity and $\mathcal{K}_{p}$ is the tablet permeability. The latter was estimated from the modified Kozeny-Carman equation (Civan, 2011):

$$
\mathcal{K}_{p}=\frac{d_{p}^{2} \varphi_{0}^{3}}{180\left(1-\varphi_{0}\right)^{2}}\left(1-\frac{\epsilon_{p}}{\varphi_{0}}\right)^{2}
$$

where $\varphi_{0}$ is the tablet porosity before any suspension gets absorbed and $d_{p}$ is the average pore diameter of the tablet. Eq. (38) does not account for the variation of the permeability with the temperature, because this is negligible compared to the variation induced by particle retention. The tablet porosity is calculated at any time by:

$$
\varphi=\varphi_{0}-\epsilon_{p}
$$

We calculated $\epsilon_{p}$ from the mass balance equation for the mixture over a differential volume containing only the pore walls. The particles being incompressible, this equation reads:

$$
\partial_{t} \epsilon_{p}=\Gamma
$$

where $\Gamma$ represents the volume rate of particles depositing on the pore walls per unit bulk volume of porous medium. The constitutive equation for $\Gamma$ will be discussed later on.

To calculate the mixture pressure inside the tablet $p_{p}$, we substituted Darcy's equation into the mass balance for the suspension over a differential control volume containing only the bulk volume of the pores, but not the pore wall, which reads:

$$
\partial_{t}(\varphi \rho)=-\partial_{\boldsymbol{x}} \cdot\left(\varphi \rho \boldsymbol{u}_{p}\right)-\rho_{s} \Gamma
$$

and obtained:

$$
\partial_{t}(\varphi \rho)=\partial_{x} \cdot\left[\left(\mathcal{K}_{p} / \eta\right) \rho \partial_{x} p_{p}\right]-\rho_{s} \Gamma
$$

For $p_{p}$, one can write two boundary conditions; one at the tablet core surface and one at the wetting front depth:

$$
p_{p}=p \text { at } z=0 \quad ; \quad p_{p}=p_{c} \text { at } z=h_{p}
$$

where $p_{c}$ is the capillary pressure and $h_{p}$ is the wetting front depth inside the tablet (Fig. 5). The capillary pressure depends on the dimensions of the average pore and the solid-liquid contact angle $(\vartheta)$ and is equal to the Laplace pressure in the capillary, given by:

$$
p_{c}=-\left(4 / d_{p}\right) \gamma \cos \vartheta
$$




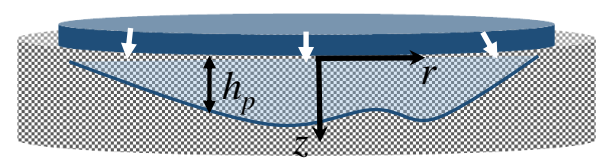

Figure 5: Wetting front depth inside the tablet.

To calculate $h_{p}$, we employed the function $F_{p}(r, z, t) \equiv h_{p}(r, t)-z$ (Leal, 2007). The coordinates $(r, z)$ of the points belonging to the wetting front surface satisfy the condition $F_{p}(r, z, t)=0$. Consequently, the substantial derivative of $F_{p}$ along the pathlines of these points is zero:

$$
D_{t} F_{p}=0 \Rightarrow \partial_{t} F_{p}+\boldsymbol{u}_{p} \cdot \partial_{\boldsymbol{x}} F_{p}=0
$$

In Eq. 45 , if we express $F_{p}$ in terms of $h_{p}$, we obtain a differential equation that allows calculating the latter:

$$
\partial_{t} h_{p}+\left.v_{p}\right|_{z=h_{p}} \partial_{r} h_{p}-\left.w_{p}\right|_{z=h_{p}}=0
$$

where $v_{p}$ and $w_{p}$ are the radial and vertical velocity components of the mixture inside the porous medium, respectively. To calculate these velocity components, one needs to express Darcy's equation (Eq. 37) in cylindrical coordinates. At $t=0$, the wetting front depth is zero, namely the tablet core is dry. At $r=0$, the gradient of $h_{p}$ vanishes by symmetry, and at the edges of the wetted area it is $h_{p}=0$.

The volume fraction of particles in the suspension is given by the mass balance equation for the suspended particles (solid phase) over a differential control volume containing only the bulk volume of the pores. This equation reads:

$$
\partial_{t}\left(\varphi \phi_{p}\right)=-\partial_{\boldsymbol{x}} \cdot\left(\varphi \phi_{p} \boldsymbol{u}_{p}\right)-\partial_{\boldsymbol{x}} \cdot \boldsymbol{j}_{p}-\rho_{s} \Gamma
$$

where $\boldsymbol{j}_{p}$ is the particle migration flux. Following Civan (2011), this is given by:

$$
\boldsymbol{j}_{p}=-\varphi \mathcal{D}_{p} \partial_{\boldsymbol{x}} \phi_{p}
$$

where $\mathcal{D}_{p}$ is the coefficient of dispersion of suspended particles migrating in the mixture. According to Civan (2011), an appropriate empirical relation that relates $\mathcal{D}_{p}$ with the Brownian diffusion coefficient was derived by Hiby (1962):

$$
\frac{\mathcal{D}_{p}}{\mathcal{D}}=0.67+\frac{0.65 \mathrm{Pe}_{\mathrm{g}}}{1+6.7 \mathrm{Pe}_{\mathrm{g}}^{-1 / 2}}, \quad \text { for } \quad 0.01 \leq \mathrm{Pe}_{\mathrm{g}} \leq 100
$$

where $\mathrm{Pe}_{\mathrm{g}}$ is the Peclet number based on the porous media mean grain diameter $\left(d_{g}\right)$ defined as $\mathrm{Pe}_{\mathrm{g}} \equiv u_{p} d_{g} / \mathcal{D}$, where $u_{p}$ denotes the order of magnitude of the velocity field of the mixture into the pores, and $\mathcal{D}$ is given by Eq. (26). According to Sarkar and Wassgren (2009), the mean diameter of grains (powder) used to create pharmaceutical tablets is $1 \mathrm{~mm}$.

In our model, to calculate the particle volume fraction inside the porous tablet we combined Eqs. (41) and (47). The resulting equation reads:

$$
\rho \varphi \partial_{t}\left(\phi_{p} / \rho\right)=-\rho \varphi \boldsymbol{u}_{p} \cdot \partial_{\boldsymbol{x}}\left(\phi_{p} / \rho\right)-\partial_{\boldsymbol{x}} \cdot \boldsymbol{j}_{p}-\left[1-\left(\rho_{s} / \rho\right) \phi_{p}\right] \Gamma
$$

At the surface of the tablet, we set $\phi_{p}=\phi$, where $\phi$ is given by Eq. (24). Additionally, at the wetting front $\left(F_{p}=0\right)$, the particle migration flux normal to the boundary (wetting front) is zero. The last boundary condition does not allow particles to migrate outside of the wetted tablet core. 
Iwasaki (1937) derived an expression for the particle retention rate on pore walls assuming this to be proportional to the magnitude of the total particle flux:

$$
\Gamma=\mathscr{F}_{p}\left|\varphi \phi_{p} \boldsymbol{u}_{p}+\boldsymbol{j}_{p}\right|
$$

where $\mathscr{F}_{p}$ is the particle "filter" coefficient. Following Elimelech et al. (2013), we estimated $\mathscr{F}_{p}$ from:

$$
\mathscr{F}_{p}=(3 / 2)\left[\left(1-\varphi_{0}\right) / d_{p}\right] \beta_{k} r_{k}\left(1-\epsilon_{p} / \epsilon_{m}\right)^{2}
$$

where $r_{k}$ denotes the single-collector contact efficiency, which represents the rate of collisions between the tablet pores and the solid particles within the coating suspension, $\epsilon_{m}$ is the maximum deposited volume fraction at which the filter coefficient becomes zero and $\beta_{k}$ is the attachment efficiency coefficient, which represents the fraction of particle collisions leading to particle attachment to the walls of the pores.

According to Elimelech et al. (2013), $\beta_{k}$ is of order one and can be determined experimentally. In this work, since we do not have any data regarding pharmaceutical suspensions, we set $\beta_{k}=1$, as assumed in Civan (2011). For different porous media $\epsilon_{m}$ is determined experimentally $\left(\epsilon_{m}=0.95 \varphi\right.$; Civan, 2011). Finally, the single-collector contact efficiency coefficient can be calculated from the empirical equation (Tufenkji and Elimelech, 2004):

$$
r_{k}=(3 / 2) \mathscr{A}_{s}\left(\frac{a}{d_{p}}\right)^{2} \quad ; \quad \mathscr{A}_{s}=\frac{2\left(1-\lambda_{p}^{5}\right)}{2-3 \lambda_{p}+3 \lambda_{p}^{5}-2 \lambda_{p}^{6}} \quad ; \quad \lambda_{p}=(1-\varphi)^{1 / 3}
$$

Notice that the above equations are valid for particles of small radius $a\left(10^{-8} \mathrm{~m}\right.$ to $\left.10^{-9} \mathrm{~m}\right)$ and in the absence of external forces (Civan, 2011). We assumed these criteria are met in our simulations.

In this section, we presented the part of our model which deals with the coating film flow inside the tablet. From Eqs. (46) and (50) one can predict the wetting front depth and the particle volume fraction inside the porous tablet, respectively. Together with Eqs. (46) and (50), we solved the expressions for the mixture velocity (Eq. 37) and pressure (Eq. 42), and the particle retention rate (Eqs. 40, 51) and migration flux (Eq. 48). For the case study investigated in this work (absorption into a cylindrical tablet), the equations need to be expressed and solved in cylindrical coordinates. They are not reported here in component form for the sake of brevity.

\subsection{Numerical solution}

To solve numerically the equations that model the behavior of the coating suspension film on the flat surface and edges of the tablet, we first made them dimensionless using the following variables:

$$
\begin{aligned}
& h \equiv H_{0} h^{*} ; W_{a} \equiv\left(\gamma / \mu_{w}\right) W_{a}^{*} ; \dot{m}_{e} \equiv \rho_{e}\left(\gamma / \mu_{w}\right) \dot{m}_{e}^{*} ; Q_{s} \equiv Q_{s}^{*}\left(\gamma / \mu_{w}\right) R_{0} ; t \equiv H_{0}\left(\mu_{w} / \gamma\right) t^{*} \\
& j_{i} \equiv\left(\gamma / \mu_{w}\right) j_{i}^{*} ; p \equiv\left(\gamma / R_{0}\right) p^{*} ; \rho \equiv \rho_{e} \rho^{*} ; \eta \equiv \mu_{w} \eta^{*} ; r \equiv R_{0} r^{*} ; z \equiv H_{0} z^{*} ; s_{1} \equiv R_{0} s_{1}^{*}
\end{aligned}
$$

where $H_{0}$ and $R_{0}$ denote the initial maximum film thickness and the characteristic length of the tablet surface including its edges, respectively. We then solved the dimensionless versions of the equations for the film thickness profile (Eqs. 14, 31) and the solid volume fraction profile (Eqs. 24, 35) together with the necessary closure equations for the suspension density (Eq. 2), velocity (Eqs. 9, 33), pressure (Eqs. 12, 34), evaporation flux (Eq. 17), viscosity (Eq. 27-30), and particle migration flux (Eq. 25). 
Moreover, we made dimensionless the evolution equations that yield the coating suspension wetting front depth (Eq. 46) and polymer volume fraction inside the porous tablet (Eq. 50) together with the necessary closure equations for the suspension velocity and pressure inside the tablet, and the particle retention and migration flux in the tablet (Eqs. 37, 42, 51 and 48, respectively) using the following dimensionless variables:

$$
h_{p} \equiv H_{0} h_{p}^{*} ; \boldsymbol{u}_{p} \equiv\left(\gamma / \mu_{w}\right) \boldsymbol{u}_{p}^{*} ; \boldsymbol{j}_{p} \equiv\left(\gamma / \mu_{w}\right) \boldsymbol{j}_{p}^{*} ; p_{p} \equiv\left(\gamma / R_{0}\right) p_{p}^{*}
$$

The equations describing the behavior of the coating mixture inside the tablet are defined in a moving domain $\left[0<z^{*}<h_{p}^{*}\left(r^{*}, t^{*}\right)\right]$. This is because the wetting front position changes with time. It is possible to turn the integration domain into one with fixed boundaries through a suitable change of independent variables. The transformation proposed by Landau (1950) is $\xi=z^{*} / h_{p}^{*}$. Implementing this transformation in the dimensionless equation for the vertical velocity component of the mixture inside the porous medium $w_{p}^{*}$ yields:

$$
w_{p}^{*}=-\frac{1}{\varphi} \frac{\mathcal{K}_{p}}{H_{0}^{2}} \frac{1}{h_{p}^{*}} \partial_{\xi} p_{p}^{*}
$$

By using Landau's transformation the moving boundary in the above equation is fixed at $\xi=1$. The rest of the equations defined in a moving domain were treated in the same way as Eq. (54), but are not reported here for the sake of brevity. More examples of equations transformed via Landau's technique can be found in Kutluay et al. (1997) and Christodoulou et al. (2018).

In Section 2, we presented the equations of the model we developed in order to predict the film thickness, imbibition depth and coating particle volume fraction onto and into a tablet. The model can also estimate the crust formation time $t_{c r}$ required for the polymer concentration to reach the critical value at which the coating suspension film must be considered as a solid.

\section{Numerical results and validation}

In this section, we present the numerical results of the model. Because the experimental data were taken from multiple sources, we did not investigate a single case study, instead we validated parts of the model separately with experimental and theoretical studies of different researchers and CFD Volume-Of-Fluid (VOF) simulations.

In Subsection 3.1, we use experimental and numerical data from the work of Niblett et al. (2017) to validate the numerical predictions concerning the drying rate of pharmaceutical coating suspension films. In Subsection 3.2, we compare the predictions of our model for the absorption of pure liquid films into porous media with the corresponding experimental results of Léang et al. (2019), who investigated the absorption of liquids of different viscosity into porous media of different permeabilities.

Furthermore, in Subsection 3.3, we present the results of our model for the flow of a non-Newtonian suspension that initially covers a curved tablet edge and its adjacent flat neighborhood, comparing them to the results of VOF simulations performed using ANSYS Fluent (ANSYS, 2019). Our numerical results are in good agreement with the VOF results.

We were unable to find experimental data suitable to validate the part of the model that accounts for the effect of particles on the absorption of a suspension into a pharmaceutical tablet. Thus, in Subsection 3.4, we only present results of the model that showcase the influence of different process parameters on the rate of suspension absorption. We performed all the numerical calculations in gPROMS (PSE, 2019), employing the Modelbuilder modeling platform. 


\subsection{Coating film drying rate}

In this work, we have developed a model that can predict the drying rate of a coating suspension on a tablet surface. The model calculates the concentration of particles into the coating suspension as it dries, taking into account that when the particle volume fraction becomes sufficiently high $(\phi=0.67)$ the coating film can no longer be regarded as a liquid with suspended particles, but behaves as a wetted solid (wetted crust; Kiil, 2006). The model yields the wetted crust formation time $t_{c r}$ for given film-coating process conditions (temperatures, relative humidity, air flow rate). According to Niblett et al., (2017), calculating this time is important since for $t>t_{c r}$ no coating suspension can be exchanged among tablets as they move (rotate) in the drum and thus no tablet defects (inter-tablet coating variability) can be created.

In this subsection, we show the ability of our model to calculate the drying rate of an aqueous pharmaceutical coating suspension (Aquarius Prime, $10 \% \mathrm{w} / \mathrm{w}$ solid particles, $\rho_{s}=1438 \mathrm{~kg} / \mathrm{m}^{3}$, $\gamma=0.046 \mathrm{~N} / \mathrm{m})$. The viscosity and density of the gas inside the drum were set to $\rho_{g}=1.092 \mathrm{~kg} / \mathrm{m}^{3}$ and $\mu_{g}=2 \cdot 10^{-2} \mathrm{cP}$, respectively. We compared the results of our model with the findings of Niblett et al. (2017). They performed experiments to study the drying rate of pharmaceutical suspensions and its effect on tablet appearance. Based on their experimental observations, they developed a model that can estimate the drying rate of the coating suspensions used in the experiments. They reported that the experiments and their model are in good agreement but did not explicitly report the experimental values for the evaporation flux nor the overall drying time. Thus, we validated our model with the equivalent numerical results reported in their work.

The tablet in both this study and that of Niblett et al. (2017) was taken to be cylindrical ( $7 \mathrm{~mm}$ in diameter). The suspension film was also considered to be approximately cylindrical and cover only the "upper" round surface of the tablet $\left(L_{s}=7 \mathrm{~mm}\right)$. Since Niblett et al. (2017) did not report initial temperatures of the tablet or of the suspension after application, we considered that the tablets are preheated close to the temperature of the bulk air (Möltgen et al., 2012) and that the suspension is sprayed at $25^{\circ} \mathrm{C}$. Finally, because Niblett et al. (2017) modeled the coating suspension behavior on flat, impermeable substrates and thus did not simulate the coating suspension flow or absorption into the tablet, we neglected these processes (for this case study only) and validated here the part of the model related to coating suspension drying presented in Section 2.1.

In Table 1, we compare the carrier fluid (water) evaporation flux $\dot{m}_{e}$ predictions of our model to those estimated by the model of Niblett et al. (2017). The conditions (drying gas velocity $v_{g}$, relative humidity $R H$, and temperature $T_{\infty}$ ) of the coating runs and simulations performed by Niblett et al. (2017) appear in Table 1 as well. These conditions were used in our validation simulations. From the model output we can deduce that higher temperature and air flow rate in the coating drum as well as lower relative humidity lead to larger evaporation rates. The model agrees well with equivalent numerical data by Niblett et al. (2017) as seen also in Figure $6(\mid \%$ Error $\mid<12 \%)$. In this figure, the dotted lines represent the $10 \%$ error lines.

Table 1: Current model predictions for coating carrier fluid evaporation mass flux $\dot{m}_{e}$. Validation with numerical results from the model of Niblett et al. (2017).

\begin{tabular}{ccccc}
\hline $\begin{array}{c}\text { Bulk gas } \\
\text { temperature }\left({ }^{\circ} \mathrm{C}\right)\end{array}$ & $\begin{array}{c}\text { Relative } \\
\text { humidity }\end{array}$ & $\begin{array}{c}\text { Gas velocity } \\
(\mathrm{m} / \mathrm{s})\end{array}$ & \multicolumn{2}{c}{$\begin{array}{c}\text { Evaporation mass flux }\left(\mathrm{kg} / \mathrm{m}^{2} \mathrm{~s}\right) \\
\text { Niblett et al., 2017 }\end{array}$} \\
\hline 60 & 0.50 & 0.20 & 0.0075 & 0.0074 \\
70 & 0.42 & 0.22 & 0.0139 & 0.0134 \\
60 & 0.58 & 0.16 & 0.0065 & 0.0060 \\
65 & 0.40 & 0.15 & 0.0084 & 0.0092 \\
65 & 0.40 & 0.15 & 0.0096 & 0.0101 \\
\hline
\end{tabular}




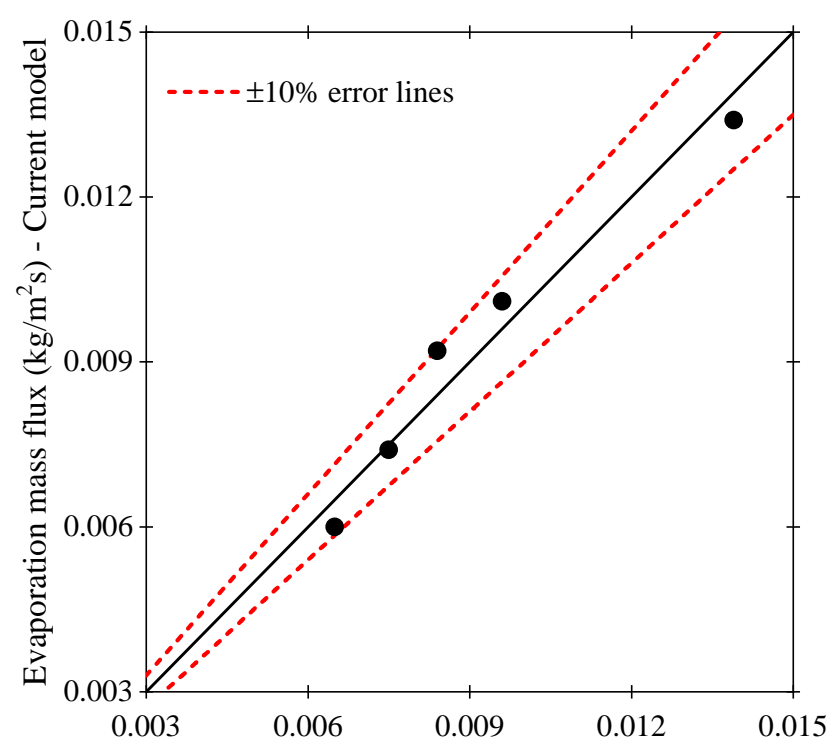

Evaporation mass flux $\left(\mathrm{kg} / \mathrm{m}^{2} \mathrm{~s}\right)$ - Niblett et al., 2017

Figure 6: Validation of numerical results for pharmaceutical coating (Aquarius Prime) drying rate. Comparison with data by Niblett et. al. (2017).

\subsection{Pure solvent absorption rate}

In this subsection, we validate our model predictions for the absorption of pure liquids into porous media with the corresponding experimental results of Léang et al. (2019). We were unable to find experimental data to validate the part of our model that predicts the absorption of a coating suspension inside a pharmaceutical tablet. Thus, we only show here the ability of the model to simulate the absorption of pure liquid films (of ranging viscosity) into different porous substrates while the films evaporate. Coating suspension penetration into porous media and the influence of particle retention on the absorption rate are discussed in Subsection 3.4.

In Figure (7.a), we compare the model predictions for the maximum water $\left(M_{w}=18 \mathrm{~g} / \mathrm{mol}\right.$, $\left.\rho=998 \mathrm{~kg} / \mathrm{m}^{3}, \mu_{w}=1 \mathrm{cP}, \gamma=0.072 \mathrm{~N} / \mathrm{m}\right)$ penetration depth into a porous medium with the corresponding experimental data of Léang et al. (2019). In the simulation and experiment, the initial film thickness, the porosity and the pore radius of the substrate were $h_{\max }=200 \mu \mathrm{m}, \varphi=0.33 \pm 0.02$ and $r_{p}=4.8-5 \mathrm{~nm}$, respectively. The water contact angle $\vartheta$ with the porous substrate was measured by Léang et al. (2019) to be $31 \pm 2^{\circ}$. The values of the relative humidity $(R H=54 \pm 2 \%)$ and the air, film and substrate temperatures $\left(20^{\circ} \mathrm{C}\right)$ used in the simulations were the same as those used in the experiments. The water evaporation mass flux reported by Léang et al. $\left(\dot{m}_{e}=4.9 \cdot 10^{-5} \mathrm{~kg} / \mathrm{m}^{2} \mathrm{~s}\right)$ was very close to that calculated by our model $\left(\dot{m}_{e}=5.01 \cdot 10^{-5} \mathrm{~kg} / \mathrm{m}^{2} \mathrm{~s}\right)$.

In Figure (7.b), we validate the model predictions for viscous liquid absorption into a porous medium (glycerol, $M_{w}=92 \mathrm{~g} / \mathrm{mol}, \rho=1260 \mathrm{~kg} / \mathrm{m}^{3}, \mu=1500 \mathrm{cP}, \gamma=0.063 \mathrm{~N} / \mathrm{m}$ ). In the simulation and experiment, the initial film thickness, the porosity and the pore radius of the substrate were $h=200 \mu \mathrm{m}, \varphi=0.31 \pm 0.03$ and $r_{p}=7.9-8 \mathrm{~nm}$, respectively. The water contact angle $\vartheta$ with the porous substrate was measured by Léang et al. (2019) to be $20 \pm 2^{\circ}$. Again, the values of the relative humidity $(R H=54 \pm 2 \%)$ and the air, film and substrate temperatures $\left(20^{\circ} \mathrm{C}\right)$ used in the simulations were the same as those used in the experiments. The absorption of glycerol (Fig. 7.b) is significantly slower than the one of water (Fig. 7.a). This is due to the high viscosity of glycerol that is inversely proportional to the flow in the pores (see Eq. 37). The results in Figures (7.a) and (7.b) are in reasonable agreement with the experimental data $(\mid \%$ Error $\mid<15 \%)$ given the uncertainty of the model input/experimental parameters. 

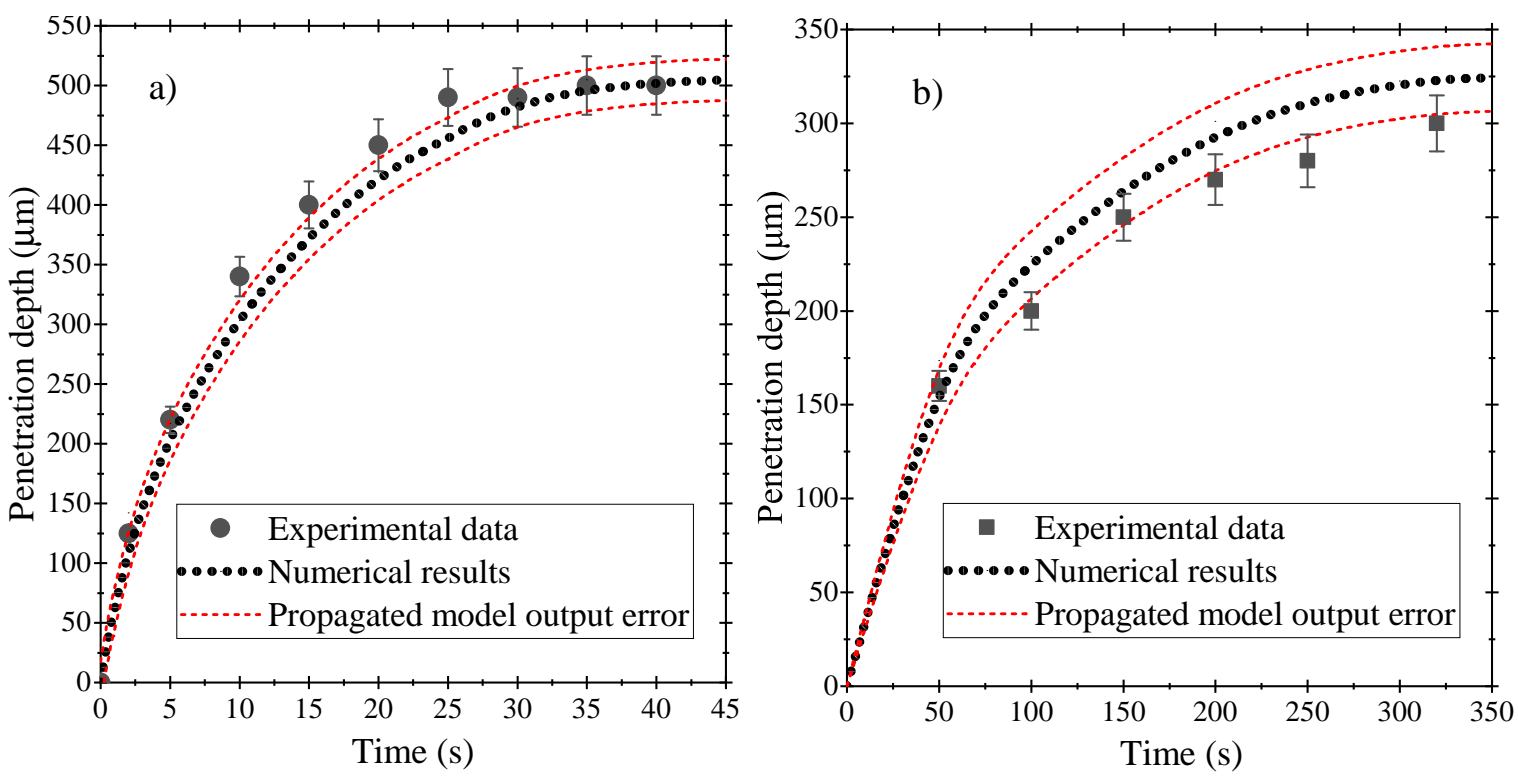

Figure 7: Validation of numerical results for pure liquid (7a. water, 7b. glycerol) absorption rate into porous substrates. Comparison with experimental data reported by Léang et al. (2019).

The red dotted lines in Figure 7 represent the propagated error due to input parameter uncertainty. Some of the input parameters of our model were difficult to determine precisely from the experimental results reported in the literature. To account for this, we propagated the experimental measurement errors (reported by Léang et al., 2019) of the input parameters to the numerical results of the model following the stochastic sampling method of Cacuci and Cacuci (2003). First, we defined the probability distributions (measurement error) of the input parameters based on experimental data we found in the literature. Subsequently, we used these distributions to generate a sample and create multiple scenarios using gPROMS Modelbuilder. Lastly, we performed a series of simulations and we calculated the standard deviation of the response variable, which in our case is the maximum wetting front depth (penetration depth). The above method is made possible by the ability of the current model to predict the wetting front in a reasonably short time $(<1 \mathrm{~min})$. Such analysis would be impossible with more computationally expensive CFD simulations.

In the case of pharmaceutical coating dispersion, the solid particles can hinder carrier fluid absorption by clogging the pores and reducing the tablet permeability. It is reported in the literature that water penetrates into the porous core (Möltgen et al., 2012) and that coating suspension absorption into the tablet promotes film adhesion (Muliadi and Sojka, 2010). Thus, we investigate qualitatively the effect of particle retention on coating absorption in Subsection 3.4.

\subsection{Coating film flow over a tablet edge}

Pharmaceutical tablets can have different shapes (round biconvex, cylindrical, oval etc.) and surfaces of different curvature. In this subsection, we study coating suspension flow in the special case where the coating suspension film covers initially a tablet edge as well as the adjacent surfaces of negligible curvature. According to O'Brien and Schwartz (2002), the thin film approximation for flow along a curved substrate, followed in this work, is still valid provided the film thickness is much less than the radii of curvature of the tablet $(h / R<0.1)$.

It is reported in the literature (Ketterhagen, 2010; Freireich et al., 2015), that less coating suspension per unit area is applied on the tablet edges than on the flat surfaces. This is due to the tablet orientation when it passes under a spray. This means that the probability of coating thickness variability is high in these tablet regions. Thus, in order to avoid defects, it is important to model the flow of a coating film, which is applied on the tablet edges. 

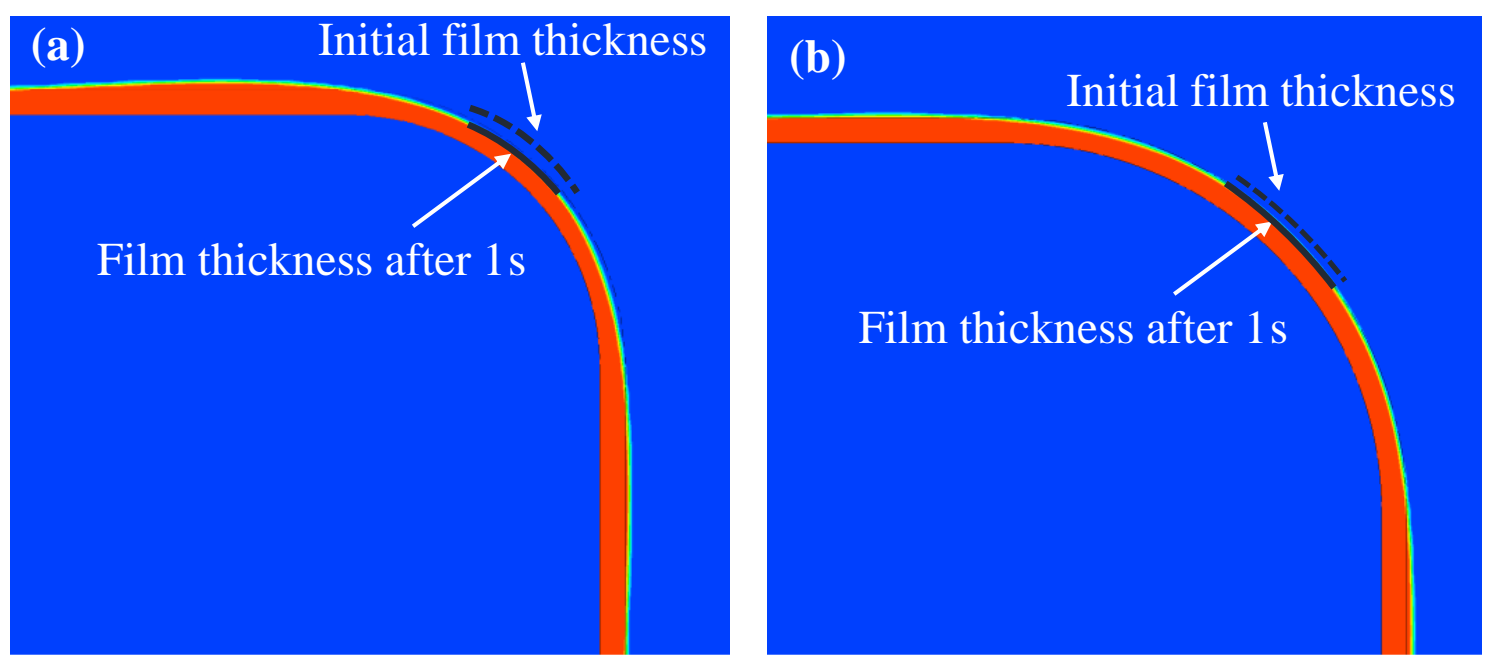

Figure 8: CFD Volume-Of-Fluid simulation of a coating film applied over a curved tablet edge. a) Radius of curvature $0.1 \mathrm{~cm}, \mathrm{~b})$ Radius of curvature $0.2 \mathrm{~cm}$.

We performed detailed two-dimensional CFD Volume-Of-Fluid (VOF) simulations and compared the results with the current model predictions for thin film flow over surfaces of different curvatures. In all the simulations, the coating suspension film was initially assumed to cover a tablet edge and the adjacent surfaces of negligible curvature (Fig. 8). To highlight the effect of tablet curvature on the film flow, we set the drying and absorption rates of the coating suspension, which were investigated in previous sections, to zero. Since no significant liquid phase evaporation occurs, we considered that the polymer particle volume fraction $\phi$ is constant. The coating suspension was assumed to behave as a non-Newtonian power law fluid $\left(n=0.90, m=0.5 \mathrm{Pas}^{n}\right)$. The initial film thickness covering all the surfaces was set to be $100 \mu \mathrm{m}$.

In Figure 8, we show the film thickness $0.1 \mathrm{~s}$ after its application on a curved surface. The film thickness was calculated by VOF simulations employing ANSYS Fluent (ANSYS, 2019). As it was expected based on previous work (Schwartz and Weidner, 1995; Weidner et al., 1996), the thickness over the edge reduces significantly compared to the initial value. In Table 2 , we compare the results for the minimum film thickness along the edge and the film thickness directly outside the edge (where the curvature is negligible compared to the edge) between our model and the VOF simulations. The results are in good agreement $(\mid \%$ Error $\mid<15 \%)$. It should be noted that the computational cost of our mathematical model is significantly smaller (CPU time $<1 \mathrm{~min}$ ) than the VOF simulations (CPU time $\approx 2$ days).

From both the current model and the VOF calculations, we can deduce that the intra-tablet coating thickness variability is significant $\left(h_{\min }<<h_{\max }\right)$. To conclude, tablet edges are not only sprayed less compared to the flat tablet surfaces (Freireich et al., 2015), but also do not "hold" the coating that is applied on them, thus leading to intra-tablet coating non-uniformity.

Table 2: Tablet edge curvature effect on coating film thickness after $0.1 \mathrm{~s}$.

\begin{tabular}{|c|c|c|c|c|}
\hline & \begin{tabular}{||c} 
Edge curvatur \\
Current model
\end{tabular} & $\begin{array}{l}\text { re radius } 0.1 \mathrm{~cm} \\
\text { CFD simulation }\end{array}$ & $\begin{array}{r}\text { Edge curvatu } \\
\text { Current model }\end{array}$ & $\begin{array}{l}\text { re radius } 0.2 \mathrm{~cm} \\
\mathrm{CFD} \text { simulation }\end{array}$ \\
\hline Min $h$ along the edge $(\mu \mathrm{m})$ & 63 & 60 & 78 & 80 \\
\hline$h$ outside the edge $(\mu \mathrm{m})$ & 118 & 120 & 111 & 110 \\
\hline
\end{tabular}




\subsection{Coating flow, absorption and evaporation}

In this subsection, we present numerical results of the overall model that highlight the influence of different process and suspension parameters on film flow and absorption into the tablet. Understanding how different parameters affect the amount of water and solid particles entering the tablet can be used to optimize the final product. Excess water inside the tablet may lead to shorter tablet shelf-life, whereas lack of particle penetration into the pores can lead to poor film adhesion (Cole et al., 1995).

Figure 9 shows the model (Section 2) predictions for the thickness and particle volume fraction profiles of a suspension film which is applied onto a tablet. In the simulation, the thin film $\left(h_{(t=0)}=100 \mu \mathrm{m}\right)$ was considered to initially cover part of the upper surface of a cylindrical tablet $1 \mathrm{~cm}$ in radius (wetted area radius is $0.8 \mathrm{~cm}$ ). The coating suspension drying, flow over the dry parts of the tablet surface, and penetration into the tablet were simulated. The temperature and relative humidity of the gas above the film were set to $T_{\infty}=50^{\circ} \mathrm{C}$ and $R H=0.50$, respectively. The tablet was assumed to be at the same temperature as the gas. The temperature profile inside the tablet during the simulation is shown in 12 in the Appendix (Section A3). These conditions are representative of the conditions in a coating process. The coating suspension was considered to have an initial volume fraction of particles of 0.20 , an initial temperature of $25^{\circ} \mathrm{C}$, and to exhibit a shear thinning behavior. For the range of shear rates in this simulation, it was regarded that the coating suspension behaves as a power law fluid. The flow and consistency indices were calculated from the experimental data of Bolleddula et al. (2010) for OpadryII White pharmaceutical suspensions: $n(\phi)=0.85 \phi^{-0.04}, m(\phi)=\hat{m}(1-\phi / 0.67)^{-2}$ where $\hat{m}=10 \mathrm{Pas}^{n}$ and $\phi \geq 0.20$. Finally, the initial tablet porosity and temperature were set to 0.3 and $50^{\circ} \mathrm{C}$, respectively, and the suspension particle size to be smaller than the pore radius $\left(a=10^{-9} \mathrm{~m}<<d_{p}=10^{-6} \mathrm{~m}\right)$.

As seen in Figure (9.a), after $7 \mathrm{~s}$ the coating suspension has spread over the dry parts of the tablet. The model predicted that the wetted area radius expands over the dry surface by $0.015 \mathrm{~cm}$. Due to the high viscosity of OpadryII suspensions the film does not spread a lot as it dries. According to our numerical results film spreading is very important over the curved edges of the tablet (see Section 3.3). Moreover, the model calculated that after $7 \mathrm{~s}$ the maximum coating suspension penetration is $55 \mu \mathrm{m}$. Figure (9.b) shows the solid particle volume fraction inside the suspension film after $7 \mathrm{~s}$. The concentration of particles is higher towards the film contact line where there is less carrier fluid as the coating film flows over its edge, shifting the latter further along the tablet surface. This was expected based on the work of Pham and Kumar (2019) who investigated the formation of coffee-ring patterns during the evaporation and imbibition of droplets containing solutes.
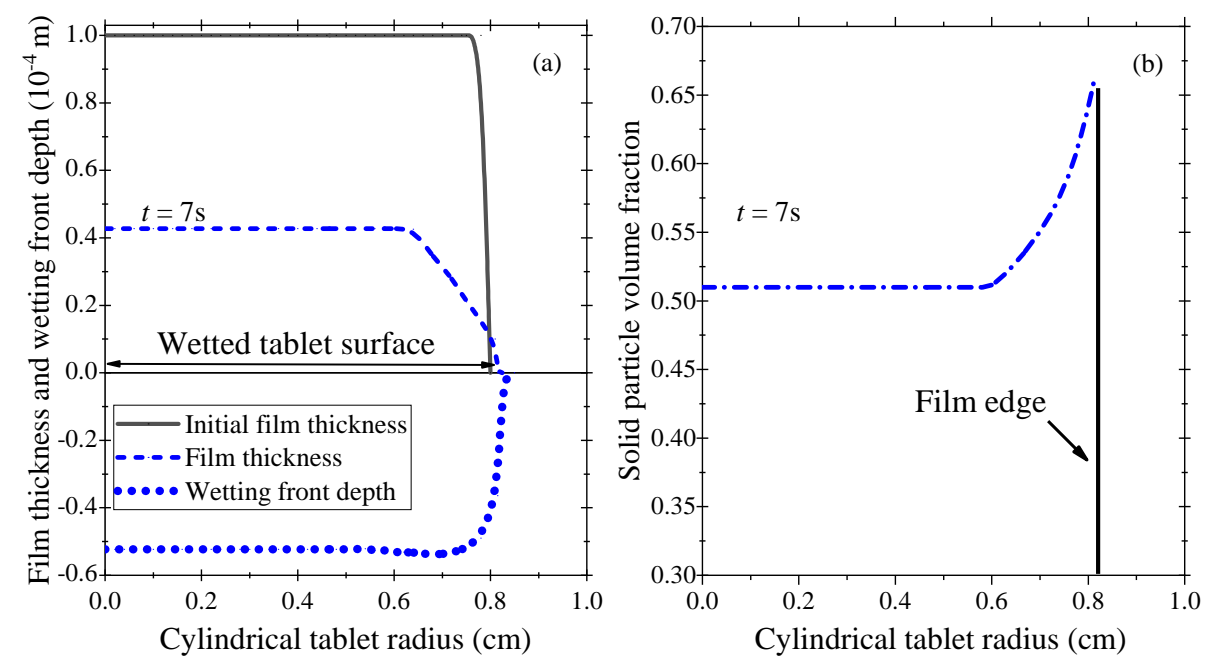

Figure 9: a) Suspension film thickness and penetration depth profiles, and b) particle volume fraction distribution in the film after $7 \mathrm{~s}$. 
In Figure (10.a), we study how the coating suspension wetting front depth is influenced by the water evaporation velocity $\left(E \equiv \dot{m}_{e} / \rho_{e}\right)$. We set different process conditions (temperatures, drying air velocity and $R H)$ so that the evaporation velocity ranges from $5 \cdot 10^{-6} \mathrm{~m} / \mathrm{s}$ to $5 \cdot 10^{-8} \mathrm{~m} / \mathrm{s}$. The initial particle volume fraction of the suspension and the initial porosity and pore radius of the tablet were the same in all simulations $\left(\phi_{0}=0.15, \varphi_{0}=0.33, d_{p}=10^{-6} \mathrm{~m}\right)$. In all case studies presented in Figure 10, the particle size was taken to be smaller than the pore radius $\left(a / d_{p}<10^{-2}\right)$ and the initial film thickness was taken to be $100 \mu \mathrm{m}$. The numerical results suggest that higher evaporation velocities lead to less suspension in the tablet. This is expected since more coating dries on the surface when the evaporation rate is high. Notice that when the evaporation velocity is significantly lower than the absorption velocity (calculated here: $W_{a} \sim 10^{-6} \mathrm{~m} / \mathrm{s}$ ) the effect of evaporation is small.

Figure (10.b) shows the effect of the initial solid volume fraction on the wetting front depth. The coating suspension was considered to be Newtonian and the initial porosity and pore diameter of the tablet as well as the evaporation velocity were the same in all simulations $\left(\varphi_{0}=0.33, d_{p}=10^{-6} \mathrm{~m}\right.$, $E=5 \cdot 10^{-6} \mathrm{~m} / \mathrm{s}$ ). Since the volume fraction of particles is connected to the viscosity of the coating suspension, higher initial concentration of particles leads to higher viscosity and thus the film spreads and penetrates into the tablet more slowly. Slower absorption means that more coating dries on the surface of the tablet and therefore the final wetting front depth is lower in the case of high initial particle volume fraction in the suspension.
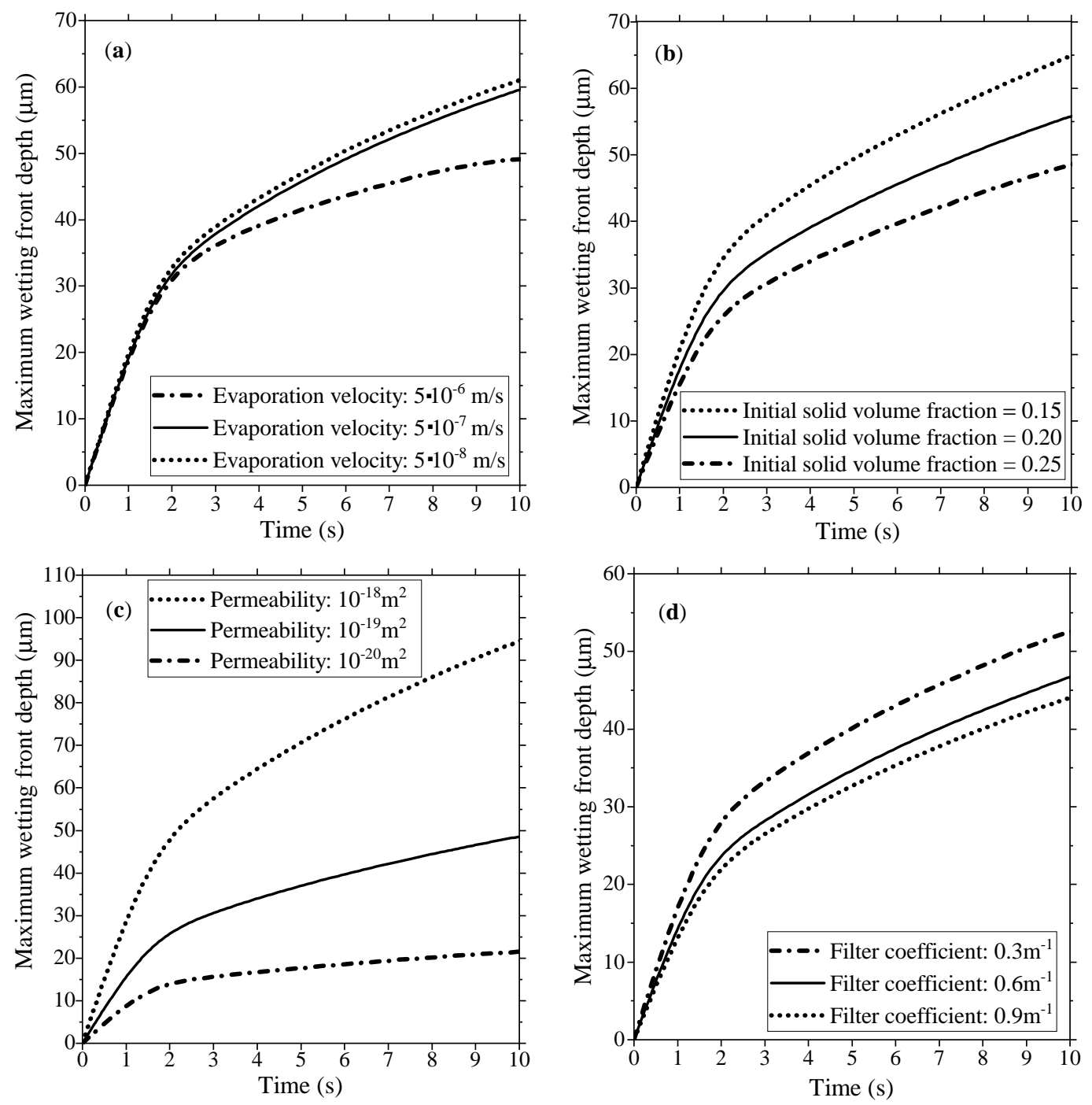

Figure 10: Effect of the a) evaporation velocity, b) initial solid volume fraction, c) tablet initial permeability, and d) filter coefficient on the coating suspension absorption rate. 
In Figure (10.c), we present numerical results of our model that highlight the influence of tablet initial permeability on coating suspension absorption into the tablet. In these simulations the evaporation velocity was calculated to be $E=5 \cdot 10^{-6} \mathrm{~m} / \mathrm{s}$ and the particle radius and initial volume fraction were taken to be $10^{-8} \mathrm{~m}$ and 0.15 , respectively. It is observed that lower initial permeability makes the effect of particle retention on the absorption rate stronger. As it was expected, the model predicts that higher initial permeability leads to more suspension into the tablet.

Particle retention can affect coating absorption by blocking tablet pores and reducing permeability. The filter coefficient $\mathscr{F}_{p}$ used in all case studies presented before was estimated from Eq. (52). Since Eq. (52) is not validated for the specific case concerning this work, we investigated the effect of the initial filter coefficient on the suspension penetration depth prediction. In Figure (10.d), we show that increasing the filter coefficient increases the volume fraction of retained particles $\left(\epsilon_{p}\right)$ and thus hinders coating absorption. The coating suspension was considered to have an initial particle volume fraction of $\phi_{0}=0.15$ and the initial porosity and pore diameter of the tablet as well as the evaporation velocity were the same in all simulations $\left(\varphi_{0}=0.33, d_{p}=10^{-6} \mathrm{~m}, E=5 \cdot 10^{-6} \mathrm{~m} / \mathrm{s}\right)$. Estimating accurately $\mathscr{F}_{p}$ is important, because it allows predicting the particle retention inside the tablet more accurately. In all case studies presented in Figure 10, the wetting front grows initially linearly in time and then sub-linearly at later times. This behavior is mainly due to the particle retention in the pores which hinders absorption.

In Figure 11, we study how the particle diameter/pore diameter ratio affects coating absorption. In the first case, we have $2 a / d_{p}=0.06$, whereas in the second $2 a / d_{p}=0.01$. The initial tablet porosity and pore diameter of the tablet as well as the water evaporation velocity were the same in both simulations $\left(\varphi_{0}=0.30, d_{p}=10^{-7} \mathrm{~m}, E=5 \cdot 10^{-6} \mathrm{~m} / \mathrm{s}\right)$. From the figure, we can observe that if $2 a / d_{p} \geq 0.06$ significant particle retention can hinder absorption after $3 \mathrm{~s}$. After this stage, the particle volume fraction in the pores is too high and the tablet becomes almost impermeable $\left(\varphi=\varphi_{0}-\epsilon_{p} \rightarrow 0\right)$. According to Holloway et al. (2011), the critical particle diameter/pore diameter ratio at which a concentrated suspension clogs a capillary tube is 0.06 . The model predicts that indeed in this case clogging occurs after a few seconds of absorption $(3 \mathrm{~s})$. Low water content and solid particles retention close to the tablet surface (as in the case study where $2 a / d_{p}=0.06$ ) are welcome by the pharmaceutical industry, because they increase the tablet shelf-life and avoid defects such as peeling of the dry coating film (Cole et al., 1995; Muliadi and Sojka, 2010).

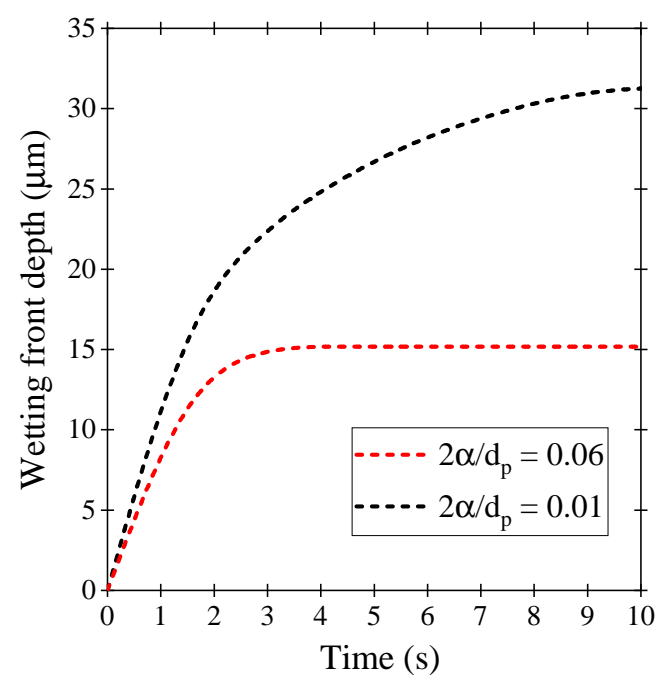

Figure 11: Effect of the particle diameter/pore diameter ratio on the suspension wetting front depth. 


\section{Conclusions}

Film-coating, a process widely used by the pharmaceutical industry, is complex to simulate accurately with reasonable computational cost. In this work, we developed a mathematical model that can quickly simulate the flow and evaporation of the coating suspension over tablet surfaces and edges, and its absorption into porous tablets. This information allows predicting the evolution of the film penetration depth inside the porous structure of the tablets and the time required for the formation of a porous crust on the tablets after a pass under the coating spray. The detailed derivations of the main equations of our mathematical model are presented in the main article and in the appendix. The results of the model, which was implemented in the gPROMS Modelbuilder platform, were validated with experimental and numerical data found in the literature and CFD Volume-Of-Fluid simulations. In Section 3, we showed that the model predictions agree well with the experiments and computationally expensive CFD numerical results.

Pharmaceutical coating film behavior on a tablet surface is affected by many parameters. To find out which ones affect the process more, we ran the model multiple times. Simulating many scenarios was possible owing to the short time required by a single run (CPU time $<1 \mathrm{~min}$ ). Indeed, an advantage of our model when compared to traditional CFD models is the computational speed. The numerical results suggested that an increase in the drying rate and initial particle concentration of the coating suspension leads to reduced coating penetration into the tablet and so less water content in the pores. Finally, we investigated the influence of the particle retention rate on the tablet permeability, finding that particles can hinder the absorption of the coating suspension into the tablet, thus affecting the amount of water and solid polymer that penetrates into the tablet.

This model can assist the pharmaceutical industry to understand the coating process better. Moreover, by predicting the amount of water and solids inside pharmaceutical solid dosage forms, it should assist in enhancing tablet shelf-life and dry film adhesion (Felton, 2013). 


\section{Appendix}

In this appendix, we present the derivations of the simplified linear momentum balance equation written for the coating suspension which forms a thin film onto the tablet (Eq. 5), the film thickness evolution equation (Eq. 14), the energy equation that yields the coating film temperature (Eq. 21) and the particle volume fraction evolution equation (Eq. 24). Finally, we discuss the estimation of the Peclet and Stokes numbers for the flows investigated in the main article.

\section{A1. Simplified momentum balance equation}

In the main article, we use the lubrication approximation theory to simplify the linear momentum balance equation written for the coating formulation (Eq. 3). The main assumption of the lubrication approximation is that, if $R$ designates the length scale of the film in the radial direction on a round tablet surface, and $H$ designates its length scale across its thickness in the direction normal to the tablet surface, then: $H / R<<1$. Having two greatly differing length scales allows simplifying the three-dimensional problem. According to Szeri (2010), one can also neglect inertial and gravitational effects in most lubricant thin films.

In this section, we derive the simplified the linear momentum balance equation (Eq. 5) of the main article in cylindrical coordinates using the lubrication approximation theory assumptions. Note that in the above derivation we neglected all changes in the azimuthal coordinate. This is because we take advantage of the axisymmetric shape of the coating film covering the tablet surface. To the best of our knowledge, the following analysis has never been reported for a suspension (pharmaceutical coating formulation) whose density and viscosity change in time.

In the radial cylindrical coordinate Eq. (3) becomes:

$$
\rho \partial_{t} v=-\rho v \partial_{r} v-\rho w \partial_{z} v-\partial_{r} p+(1 / r) \partial_{r}\left(r \tau_{r r}\right)+\partial_{z} \tau_{z r}-\tau_{\theta \theta} / r
$$

By scaling Eq. (a1) we get:

$$
\partial_{\bar{t}} \bar{v}=-\frac{V t_{c}}{R} \bar{v} \partial_{\bar{r}} \bar{v}-\frac{W t_{c}}{H} \bar{w} \partial_{\bar{z}} \bar{v}-\frac{p_{c} t_{c}}{\rho V R} \partial_{\bar{r}} \bar{p}+\frac{\mu t_{c}}{\rho R^{2}} \frac{1}{\bar{r}} \partial_{\bar{r}}\left(\bar{r} \bar{\tau}_{r r}\right)+\frac{\mu t_{c}}{\rho H^{2}} \partial_{\bar{z}} \bar{\tau}_{z r}-\frac{\mu t_{c}}{\rho R^{2}} \frac{\bar{\tau}_{\theta \theta}}{\bar{r}}
$$

where the following scales were used:

$$
\begin{gathered}
\bar{r} \equiv r / R ; \bar{z} \equiv z / H ; \bar{w} \equiv w / W ; \bar{v} \equiv v / V ; \bar{p} \equiv p / p_{c} ; \bar{t} \equiv t / t_{c} ; \bar{\tau}_{z r} \equiv(H / \mu U) \tau_{z r} \\
\bar{\tau}_{r z} \equiv(H / \mu U) \tau_{r z} ; \bar{\tau}_{r r} \equiv(R / \mu U) \tau_{r r} ; \bar{\tau}_{z z} \equiv(H / \mu V) \tau_{r r} ; \bar{\tau}_{\theta \theta} \equiv(R / \mu U) \tau_{\theta \theta}
\end{gathered}
$$

where where $\rho$ and $\mu$ are the density and viscosity of the suspension, respectively (which, for simplicity, are taken to be constants), while $t_{c}$ and $p_{c}$ are the characteristic time and pressure scales of the flow.

In Eq. (a2) we see that various time scales arise:

$$
\tau_{c, 1} \equiv R / V ; \tau_{c, 2} \equiv H / W ; \tau_{d, 1} \equiv \rho R^{2} / \mu ; \tau_{d, 2} \equiv \rho H^{2} / \mu
$$

From the continuity equation we conclude that $\tau_{c, 1}=\tau_{c, 2}$. Additionally, comparing $\tau_{d, 1}$ and $\tau_{d, 2}$ yields: $\tau_{d, 2}=(H / R)^{2} \tau_{d, 1}<<\tau_{d, 1}$. Finally, we note that:

$$
\tau_{d, 2} / \tau_{c, 1}=\frac{H^{2} V \rho}{\mu R}=\frac{H}{R} \frac{\rho H V}{\mu}=\frac{H}{R} \operatorname{Re}
$$


Knowing that $H / R<<1$ and also $\operatorname{Re}<<1$ we conclude that the shortest characteristic time is $\tau_{d, 2}$. Substituting $t_{c} \equiv \tau_{d, 2}$ into Eq. (a2) gives:

$$
\partial_{\bar{t}} \bar{v}=-\frac{H}{R} \operatorname{Re} \bar{v} \partial_{\bar{r}} \bar{v}-\frac{H}{R} \operatorname{Re} \bar{w} \partial_{\bar{z}} \bar{v}-\frac{H}{R} \frac{p_{c} H}{\mu V} \partial_{\bar{r}} \bar{p}+\left(\frac{H}{R}\right)^{2} \frac{1}{\bar{r}} \partial_{\bar{r}}\left(\bar{r} \bar{\tau}_{r r}\right)+\partial_{\bar{z}} \bar{\tau}_{z r}-\left(\frac{H}{R}\right)^{2} \frac{\bar{\tau}_{\theta \theta}}{\bar{r}}
$$

Assuming that the pressure term is as important as the viscous term, we obtain $p_{c} \equiv(R / H)(\mu V / H)$. Substituting the pressure term in Eq. (a5) and eliminating the negligible terms yields:

$$
\partial_{\bar{t}} \bar{v}=-\partial_{\bar{r}} \bar{p}+\partial_{\bar{z}} \bar{\tau}_{z r}
$$

Let us assume that the system evolves toward steady state. This assumption is valid for a thin suspension film that is deposited on the surface of a tablet. At steady state, the pressure term is equal in magnitude to the viscous term, and their combination vanishes. Before this happens, the convective terms cease to be negligible. In fact, they start dictating the time scale. This is no longer $\tau_{d, 2}$, but becomes $\tau_{c, 1}$. In this part of the time domain, a new approximation holds, which we get by rescaling the equation of motion.

We now choose $t_{c} \equiv \tau_{c, 1}$ and Eq. (a2) becomes:

$$
\frac{H}{R} \operatorname{Re} \partial_{\bar{t}} \bar{v}=-\frac{H}{R} \operatorname{Re}\left(\bar{v} \partial_{\bar{r}} \bar{v}+\bar{w} \partial_{\bar{z}} \bar{v}\right)-\frac{H}{R} \frac{p_{c} \operatorname{Re}}{\rho V^{2}} \partial_{\bar{r}} \bar{p}+\left(\frac{H}{R}\right)^{2} \frac{1}{\bar{r}} \partial_{\bar{r}}\left(\bar{r} \bar{\tau}_{r r}\right)+\partial_{\bar{z}} \bar{\tau}_{z r}-\left(\frac{H}{R}\right)^{2} \frac{\bar{\tau}_{\theta \theta}}{\bar{r}}
$$

which reduces to:

$$
\frac{H}{R} \frac{p_{c} \operatorname{Re}}{\rho V^{2}} \partial_{\bar{r}} \bar{p}=\partial_{\bar{z}} \bar{\tau}_{z r}
$$

Since the left-hand side must have unit order of magnitude, we obtain $p_{c} \equiv(R / H)(1 / \operatorname{Re}) \rho V^{2}$ and the final leading order equation is:

$$
\partial_{\bar{r}} \bar{p}=\partial_{\bar{z}} \bar{\tau}_{z r}
$$

Written in dimensional form this yields Eq. (5a) of the main article.

In the vertical cylindrical coordinate Eq. (3) becomes:

$$
\rho \partial_{t} w=-\rho v \partial_{r} w-\rho w \partial_{z} w-\partial_{z} p+(1 / r) \partial_{r}\left(r \tau_{r z}\right)+\partial_{z} \tau_{z z}
$$

Scaling Eq. (a10) with the scales appearing in Eq. (a3) and considering again that the system evolves toward steady state and that the characteristic time is the convection characteristic time $\left(\tau_{c, 1} \equiv H / W \equiv R / V\right)$ gives:

$$
\frac{H}{R} \operatorname{Re} \partial_{\bar{t}} \bar{w}=-\frac{H}{R} \operatorname{Re}\left(\bar{v} \partial_{\bar{r}} \bar{w}+\bar{w} \partial_{\bar{z}} \bar{w}\right)-\frac{p_{c} \operatorname{Re}}{\rho V^{2}} \frac{R}{H} \partial_{\bar{z}} \bar{p}+\frac{1}{\bar{r}} \partial_{\bar{r}}\left(\bar{r} \bar{\tau}_{r z}\right)+\partial_{\bar{z}} \bar{\tau}_{z z}
$$

which reduces to:

$$
\frac{p_{c} \operatorname{Re}}{\rho V^{2}} \frac{R}{H} \partial_{\bar{z}} \bar{p}=\frac{1}{\bar{r}} \partial_{\bar{r}}\left(\bar{r} \bar{\tau}_{r z}\right)+\partial_{\bar{z}} \bar{\tau}_{z z}
$$

Substituting in Eq. (a12) the scale of the pressure $p_{c}$ obtained above for the case when the system evolves towards steady state, yields:

$$
\partial_{\bar{z}} \bar{p}=\left(\frac{H}{R}\right)^{2}\left[\frac{1}{\bar{r}} \partial_{\bar{r}}\left(\bar{r} \bar{\tau}_{r z}\right)+\partial_{\bar{z}} \bar{\tau}_{z z}\right]
$$

In the limit of $H / R<<1$, we can thus regard $\partial_{\bar{z}} \bar{p}$ equal to zero. This yields Eq. (5b) of the main article. 


\section{A2. Film thickness evolution equation}

In this section, we derive the film thickness evolution equation from the continuity equation written for the mixture. The latter in cylindrical coordinates reads:

$$
\partial_{t} \rho=-(1 / r) \partial_{r}(r \rho v)-\partial_{z}(\rho w)
$$

Integrating Eq. (a14) over the vertical direction $z$, from $z=0$ to $z=h$, gives:

$$
\int_{0}^{h} \partial_{t} \rho d z=-(1 / r) \int_{0}^{h} \partial_{r}(r \rho v) d z-\int_{0}^{h} \partial_{z}(\rho w) d z
$$

The term on the left-hand side can be written as:

$$
\int_{0}^{h} \partial_{t} \rho d z=\partial_{t} \int_{0}^{h} \rho d z-\left.\rho\right|_{z=h} \partial_{t} h=\partial_{t} \int_{0}^{h} \rho d z-\rho_{e} \partial_{t} h
$$

where we have assumed that the mixture (coating suspension) density at the film-air interface is the density of the water that evaporates. The first and second terms on the right-hand of Eq. (a15) side can be expanded as follows:

$$
\begin{gathered}
-(1 / r) \int_{0}^{h} \partial_{r}(r \rho v) d z=-(1 / r) \partial_{r} \int_{0}^{h} \rho r v d z+\left.(\rho v)\right|_{z=h} \partial_{r} h \\
-\int_{0}^{h} \partial_{z}(\rho w) d z=-\left.(\rho w)\right|_{0} ^{h}=-\left.(\rho w)\right|_{z=h}+\left.(\rho w)\right|_{z=0}
\end{gathered}
$$

Substituting Eqs. (a16) to (a18) into Eq. (a15) yields:

$$
\partial_{t} \int_{0}^{h} \rho d z-\rho_{e} \partial_{t} h=-(1 / r) \partial_{r} \int_{0}^{h} \rho r v d z+\left.(\rho v)\right|_{z=h} \partial_{r} h-\left.(\rho w)\right|_{z=h}+\left.(\rho w)\right|_{z=0}
$$

At $z=0$ the boundary does not move. So, the last term on the right hand side represents the mass that leaves the control volume. This term represents the mass loss owing to absorption. In the main article, this term is taken equal to $-\rho W_{a}$, where $W_{a}$ is the absorption velocity at the film-tablet interface. The term immediately preceding the absorption flux in Eq. (a19) cannot be regarded as the mass loss owing to evaporation $\left(\dot{m}_{e}\right)$. This is because the interface (or equivalently the boundary of the control volume) moves. However, we can use the mass jump condition and write:

$$
\dot{m}_{e}=\left.\boldsymbol{n} \cdot\left(\boldsymbol{u}-\boldsymbol{u}_{s}\right) \rho\right|_{z=h}
$$

where $\boldsymbol{n}$ is the unit vector normal to the interface pointing from the mixture into the air, $\boldsymbol{u}$ is the mixture velocity, $\boldsymbol{u}_{s}$ is the velocity of the interface and $\rho$ is the mixture density. At the interface $(z=h)$, the latter is equal to the density of the pure liquid (water) that evaporates. In cylindrical coordinates Eq. (a20) becomes:

$$
\dot{m}_{e}=\left[n_{r}\left(v_{i}-u_{s r}\right)+n_{z}\left(w_{i}-u_{s z}\right)\right] \rho_{e}
$$

where $v_{i}$ and $w_{i}$ are the radial and vertical velocity components of the mixture at the film-air interface, respectively, and $\rho_{e}$ is the density of the suspension at the interface. To proceed we need to relate $n_{r}$ and $n_{r}$ to the film thickness $h(r, t)$. To do this, we write:

$$
F=z-h(r, t) \quad ; \quad \boldsymbol{n}=\frac{\partial_{\boldsymbol{x}} F}{\left|\partial_{\boldsymbol{x}} F\right|}
$$


From Eq. (a22) one obtains:

$$
\boldsymbol{n}=-\frac{\partial_{r} h}{\sqrt{1+\left(\partial_{r} h\right)^{2}}} \hat{\boldsymbol{r}}+\frac{1}{\sqrt{1+\left(\partial_{r} h\right)^{2}}} \hat{\boldsymbol{z}}
$$

where $\hat{\boldsymbol{r}}$ and $\hat{\boldsymbol{z}}$ denote the unit vector in the radial and vertical direction, respectively. The vector of unit magnitude tangent to the film and normal to $\boldsymbol{n}$ is given by:

$$
\boldsymbol{t}=\frac{1}{\sqrt{1+\left(\partial_{r} h\right)^{2}}} \hat{\boldsymbol{r}}+\frac{\partial_{r} h}{\sqrt{1+\left(\partial_{r} h\right)^{2}}} \hat{\boldsymbol{z}}
$$

We assume that the velocity of the mixture tangent to the interface is equal to the velocity of the interface. So:

$$
\boldsymbol{u}_{i} \cdot \boldsymbol{t}=\boldsymbol{u}_{s} \cdot \boldsymbol{t} \Rightarrow v_{i} t_{r}+w_{i} t_{z}=u_{s r} t_{r}+u_{s z} t_{z}
$$

Since $t_{r}=n_{z}$ and $t_{z}=-n_{r}$ we get:

$$
\left(v_{i}-u_{s r}\right) n_{z}=\left(w_{i}-u_{s z}\right) n_{r}
$$

Substituting Eq. (a26) into the jump condition yields:

$$
\dot{m}_{e}=\left[\frac{n_{r}^{2}}{n_{z}}\left(w_{i}-u_{s z}\right)+n_{z}\left(w_{i}-u_{s z}\right)\right] \rho_{e}=\frac{1}{n_{z}}\left(w_{i}-u_{s z}\right)
$$

The vertical velocity of the interface $u_{s z}$ is given by:

$$
u_{s z}=\partial_{t} h+u_{s r} \partial_{r} h
$$

From Eqs. (a26) and (a28) we get:

$$
u_{s z}=\frac{\partial_{t} h+\left[v_{i}+\left(n_{r} / n_{z}\right) w_{i}\right] \partial_{r} h}{1-\left(n_{r} / n_{z}\right) \partial_{r} h}
$$

Since it is $n_{z}=1 / \sqrt{1+\left(\partial_{r} h\right)^{2}}$ and $n_{r} / n_{z}=-\partial_{r} h$, substituting Eq. (a29) into the jump condition (a27) yields:

$$
\rho_{e} w_{i}=\frac{1}{\sqrt{1+\left(\partial_{r} h\right)^{2}}} \dot{m}_{e}+\rho_{e} \frac{\partial_{t} h+\left(v_{i}+w_{i} \cdot \partial_{r} h\right) \partial_{r} h}{1+\left(\partial_{r} h\right)^{2}}
$$

In this work, we are interested in thin films where $\partial_{r} h<<1$. Thus, Eq. (a30) becomes:

$$
\rho_{e} w_{i}=\dot{m}_{e}+\rho_{e}\left[\partial_{t} h+v_{i} \partial_{r} h\right]
$$

Substituting the above equation into the mass balance equation (Eq. a19) gives:

$$
\partial_{t} \int_{0}^{h} \rho d z=-(1 / r) \partial_{r} \int_{0}^{h} r \rho v d z-\dot{m}_{e}-\rho W_{a}
$$

Following Weidner et al. (1996), we considered that the density of the thin film does not change significantly in the vertical direction. Assuming that the mixture density is uniform over (most of) the film thickness, one can see that Eq. (a32) reduces to Eq. (14) of the main article. 


\section{A3. Energy equation}

The equation of change for temperature reported in Bird et al. (2007) reads:

$$
\rho_{1} c_{p 1} D_{t} T_{1}=-\partial_{\boldsymbol{x}} \cdot \boldsymbol{q}_{1}+\boldsymbol{\tau}_{1}: \partial_{\boldsymbol{x}} \boldsymbol{u}_{1}-\left(\frac{\partial \ln \rho_{1}}{\partial \ln T_{1}}\right)_{p} D_{t} p_{1}
$$

where $D_{t}$ is the substantial time derivative and $c_{p}, \boldsymbol{q}, \boldsymbol{u}$ and $\boldsymbol{\tau}$ denote the heat capacity, heat flux, velocity field, and deviatoric stress tensor, respectively. Here we have used the subscript 1 to identify the mixture. The last term on the right-hand side is zero for fluids with constant density. The mixture has not constant density, but it is expected to vary very little, in particular with the temperature. The generation term (conversion of kinetic energy into internal energy) is very small and can be neglected as well. So, we write:

$$
\rho_{1} c_{p 1} D_{t} T_{1}=-\partial_{\boldsymbol{x}} \cdot \boldsymbol{q}_{1}
$$

We now integrate over the region $\mathcal{R}_{1}$ occupied by the film, which is bounded by the surfaces $\mathcal{S}_{12}$ (film-air interface) and $\mathcal{S}_{13}$ (film-tablet interface). Therefore, we write:

$$
\int_{\mathcal{R}_{1}} \rho_{1} c_{p 1} D_{t} T_{1} d \boldsymbol{x}=-\int_{\mathcal{R}_{1}} \partial_{\boldsymbol{x}} \cdot \boldsymbol{q}_{1} d \boldsymbol{x}
$$

To manipulate the term on the right-hand side, we use the divergence theorem:

$$
\int_{\mathcal{R}_{1}} \partial_{\boldsymbol{x}} \cdot \boldsymbol{q}_{1} d \boldsymbol{x}=\int_{\mathcal{S}_{12}} \boldsymbol{q}_{1} \cdot \boldsymbol{n}_{12} d \boldsymbol{x}+\int_{\mathcal{S}_{13}} \boldsymbol{q}_{1} \cdot \boldsymbol{n}_{13} d \boldsymbol{x}
$$

where $\boldsymbol{n}_{i j}$ denotes the unit vector normal to $\mathcal{S}_{i j}$ pointing from phase $i$ into phase $j$.

Since the film is very thin, to manipulate the term on the left-hand side, we assume that $T_{1}$ is uniform. Note that this assumption cannot be used for the term on the right-hand side, because if we did, the term would vanish. So, we obtain:

$$
D_{t} T_{1} \int_{\mathcal{R}_{1}} \rho_{1} c_{p 1} d \boldsymbol{x}=-\int_{\mathcal{S}_{12}} \boldsymbol{q}_{1} \cdot \boldsymbol{n}_{12} d \boldsymbol{x}-\int_{\mathcal{S}_{13}} \boldsymbol{q}_{1} \cdot \boldsymbol{n}_{13} d \boldsymbol{x}
$$

If $T_{1}$ is uniform we can write: $D_{t} T_{1}=\partial_{t} T_{1}$.

Moreover, the term $\rho_{1} c_{p 1}$ is given by:

$$
\rho_{1} c_{p 1}=\rho_{e}(1-\phi) c_{p e}+\rho_{s} \phi c_{p s}
$$

and is therefore a function of the $\phi$, which in turn is a function of $r$ and $t$.

We now define:

$$
\left\langle\rho_{1} c_{p 1}\right\rangle \equiv \frac{1}{V_{1}} \int_{\mathcal{R}_{1}} \rho_{1} c_{p 1} d \boldsymbol{x}
$$

where $V_{1}$ is the volume of the film.

The simplified enthalpy jump condition at the film-gas interface $\mathcal{S}_{12}$, reads (Delhaye, 1974):

$$
\dot{m}_{e}\left(\hat{H}_{1}-\hat{H}_{2}\right)=-\boldsymbol{n}_{12} \cdot\left(\boldsymbol{q}_{1}-\boldsymbol{q}_{2}\right)
$$

To derive an expression for $\boldsymbol{q}_{1}$ we considered that $\Delta H_{e} \equiv \hat{H}_{2}-\hat{H}_{1}$ and $\boldsymbol{n}_{12} \cdot \boldsymbol{q}_{2} \equiv h_{H}\left(T_{1}-T_{2 \infty}\right)$, where $\Delta H_{e}, h_{H}$ and $T_{2 \infty}$ denote the enthalpy of vaporization, heat transfer coefficient and bulk air temperature. 
Eq. (a40) finally becomes:

$$
\boldsymbol{n}_{12} \cdot \boldsymbol{q}_{1}=\dot{m}_{e} \Delta H_{e}+h_{H}\left(T_{1}-T_{2 \infty}\right)
$$

The simplified enthalpy jump condition written for the film-tablet interface $\mathcal{S}_{13}$ yields an expression for the heat conduction from the tablet to the film:

$$
\boldsymbol{n}_{13} \cdot \boldsymbol{q}_{1}=\boldsymbol{n}_{13} \cdot \boldsymbol{q}_{3}=\left.k_{3} \partial_{z} T_{3}\right|_{z=0}
$$

where $k_{3}$ is the heat conductivity of the tablet core and $T_{3}$ is the tablet core temperature.

Substituting Eqs. (a39), (a41) and (a42) into (a37) gives:

$$
\left\langle\rho_{1} c_{p 1}\right\rangle V_{1} \partial_{t} T_{1}=-\int_{\mathcal{S}_{12}} \dot{m}_{e} \Delta H_{e} d s-\int_{\mathcal{S}_{12}} h_{H}\left(T_{1}-T_{2 \infty}\right) d s-\left.\int_{\mathcal{S}_{13}} k_{3} \partial_{z} T_{3}\right|_{z=0} d s
$$

On the right-hand side all the integrated functions are uniform over the surfaces. Thus:

$$
\left\langle\rho_{1} c_{p 1}\right\rangle V_{1} \partial_{t} T_{1}=-\dot{m}_{e} \Delta H_{e} A_{12}-h_{H}\left(T_{1}-T_{2 \infty}\right) A_{12}-\left.k_{3} \partial_{z} T_{3}\right|_{z=0} A_{13}
$$

where $A_{12}$ and $A_{13}$ are the areas of the film-air and film-tablet interfaces respectively. We used the above energy equation (Eq. 21) in the current model to calculate the film temperature.

\section{A3. Heat conduction from the tablet to the film}

The last term in Eqs. (21) and (a44) denotes the heat conduction from the tablet core to the coating film. To estimate this term, we must calculate the temperature profile in the tablet $\left(T_{3}\right.$, seen in Figure 12) using the following equation of energy conservation:

$$
\partial_{t} T_{3}=\left[k_{3} /\left(c_{p 3} \rho_{3}\right)\right] \partial_{z z}^{2} T_{3}
$$

with boundary conditions:

$$
T_{3}=T_{1} \text { at } z=0 ; \partial_{z} T_{3}=0 \text { at } z=-\ell
$$

where $\ell$ is the distance between the tablet-film interface $(z=0)$ and the tablet core center $(\ell \sim 1 \mathrm{~mm})$, and $T_{1}$ is the temperature of the coating film $\left[\right.$ at $\left.t=0, T_{1}=25^{\circ} \mathrm{C}\right]$. The tablet density, heat capacity and thermal conductivity were taken from the literature $\left(\rho_{3}=1300 \mathrm{~kg} / \mathrm{m}^{3}, c_{p 3}=1000 \mathrm{~J} / \mathrm{kg} \mathrm{K}, k_{3}=\right.$ $0.7 \mathrm{~J} / \mathrm{s} \mathrm{m} \mathrm{K}$; Krok et al., 2017). In writing Eq. (a45), we assumed that $T_{3}$ depends only on $z$. That is, we neglected radial temperature gradients inside the tablet core, because these are significantly milder than those in the $z$ direction (especially close to the film-tablet boundary; see Fig. 12).

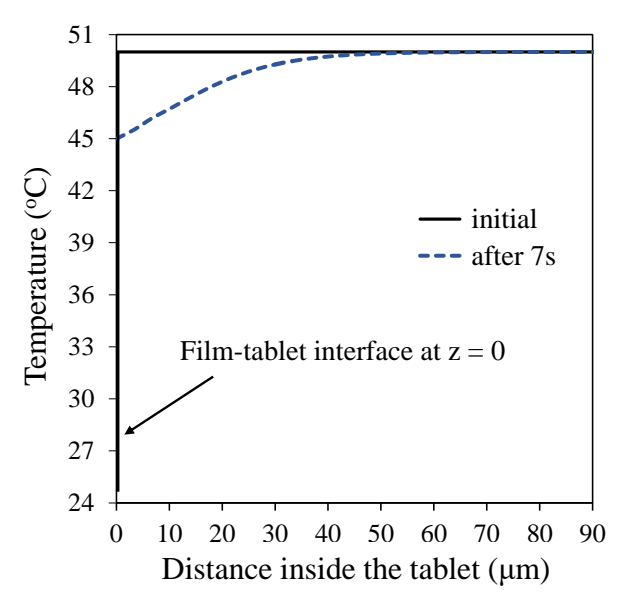

Figure 12: Temperature profile in the $\mathrm{z}$ direction inside the tablet. The case study parameters are reported in Section 3.4 (used to produce the numerical results shown in Fig. 9). 
Fig. 12 shows that $\partial_{z} T_{3}$ is significant close to the film-tablet interface and negligible in the tablet core. Away from the film-tablet interface, $T_{3}$ remains uniform during the process.

\section{A4. Particle volume fraction evolution equation}

Here, we rigorously derive a particle volume fraction evolution equation (Eq. 33) from the continuity equation written for the solid phase inside the coating film. Integrating Eq. (24) over z gives:

$$
\int_{0}^{h} \partial_{t} \phi d z=-(1 / r) \int_{0}^{h} \partial_{r}(r \phi v) d z-\int_{0}^{h} \partial_{z}(\phi w) d z-(1 / r) \int_{0}^{h} \partial_{r}\left(r j_{r}\right) d z
$$

The term on the left-hand side can be written as:

$$
\int_{0}^{h} \partial_{t} \phi d z=\partial_{t} \int_{0}^{h} \phi d z-\left.\phi\right|_{z=h} \partial_{t} h
$$

The first term on the right-hand side can be expanded as:

$$
(1 / r) \int_{0}^{h} \partial_{r}(r \phi v) d z=(1 / r) \partial_{r} \int_{0}^{h} r \phi v d z-\left.(\phi v)\right|_{z=h} \partial_{r} h
$$

The second term on the right-hand side becomes:

$$
\int_{0}^{h} \partial_{z}(\phi w) d z=\left.(\phi w)\right|_{z=h}-\left.(\phi w)\right|_{z=0}
$$

Performing the same analysis as in the Section A2 and assuming that no particles evaporate allow us to write:

$$
\left.(\phi w)\right|_{z=h}-\left.(\phi w)\right|_{z=0}=\left.\phi\right|_{z=h} \partial_{t} h+\left.(\phi v)\right|_{z=h} \partial_{r} h+\left.\phi\right|_{z=0} W_{a}
$$

Finally, the third on the right-hand side becomes:

$$
(1 / r) \int_{0}^{h} \partial_{r}\left(r j_{r}\right) d z=(1 / r) \partial_{r} \int_{0}^{h} r j_{r} d z-\left.j_{r}\right|_{z=h} \partial_{r} h
$$

Substituting Eqs. (a52), (a53), (a55) and (a56) into (a51) yields:

$$
\begin{gathered}
\partial_{t} \int_{0}^{h} \phi d z-\left.\phi\right|_{z=h} \partial_{t} h=-(1 / r) \partial_{r} \int_{0}^{h} r \phi v d z+\left.(\phi v)\right|_{z=h} \partial_{r} h \\
-\left.\phi\right|_{z=h} \partial_{t} h-\left.(\phi v)\right|_{z=h} \partial_{r} h-\left.\phi\right|_{z=0} W_{a}-(1 / r) \partial_{r} \int_{0}^{h} r j_{r} d z+\left.j_{r}\right|_{z=h} \partial_{r} h
\end{gathered}
$$

Making use of the "well mixed" approximation (uniform $\phi$ across the thickness of the film) and that the migration flux at the film-air interface are zero we get:

$$
\partial_{t}(\phi h)=-(1 / r) \partial_{r}\left(r \phi \int_{0}^{h} v d z\right)-\phi W_{a}-(1 / r) \partial_{r}\left(r h j_{r}\right)
$$

Using the film thickness evolution equation the first term on the right-hand side of the above equation can be expanded:

$$
\begin{gathered}
(1 / r) \partial_{r}\left(r \phi \int_{0}^{h} v d z\right)=(1 / r) \partial_{r}[(\phi / \rho) \rho r Q]=(1 / r)\left[\rho r Q \partial_{r}(\phi / \rho)+(\phi / \rho) \partial_{r}(\rho r Q)\right] \\
=\rho Q \partial_{r}(\phi / \rho)-(\phi / \rho) \partial_{t}(\rho h)-\phi W_{a}-\phi\left(\dot{m}_{e} / \rho\right)
\end{gathered}
$$

where $Q$ is the surface flux given by Eq. (15). 
Eq. (a55) then becomes:

$$
\partial_{t}(\phi h)=-\rho Q \partial_{r}(\phi / \rho)+(\phi / \rho) \partial_{t}(\rho h)+\phi\left(\dot{m}_{e} / \rho\right)-(1 / r) \partial_{r}\left(r h j_{r}\right)
$$

which simplifies to Eq. (24) of the main article.

\section{A5. Estimation of the Peclet and Stokes numbers}

To derive an expression for the particle migration flux in the main article, it is important to first estimate the Peclet number $\mathrm{Pe} \equiv a^{2} \dot{\gamma} / \mathcal{D}$ where $\mathcal{D}$ is the Brownian diffusion coefficient of the particles in the suspension, $a$ is the particle radius (assuming the particles are spherical) and $\dot{\gamma}$ is the shear rate scale. In this work it is $\dot{\gamma} \sim 1 \mathrm{~s}^{-1}$ and $a \sim 10^{-8} \mathrm{~m}$.

The particle Brownian diffusion coefficient $\mathcal{D}$ is estimated by Eq. (27) of the main article. The term in front of the Einstein diffusivity $\mathcal{D}_{0}$ is of order 1 for all the values of $\phi$ of interest in our simulations $\left(\phi<\phi^{*}\right)$. At high particle volume fractions (at the maximum packing limit; $\phi \rightarrow \phi^{*}$ ), the diffusivity diverges and the simulation is completed as discussed in the main article. Thus, for the Brownian diffusion coefficient it is $\mathcal{D} \sim \mathcal{D}_{0}=\left(k_{B} T_{w}\right) /\left(6 \pi \mu_{w} a\right)$ where $k_{B}=10^{-23} \mathrm{~J} / \mathrm{K}$ is the Boltzmann constant, and $T_{w} \approx 320 \mathrm{~K}$ and $\mu_{w}=0.001 \mathrm{Pas}$ are the carrier fluid temperature and viscosity, respectively. Thus, we get $\mathcal{D} \sim 10^{-10} \mathrm{~m}^{2}$.

The Peclet number can be estimated:

$$
\mathrm{Pe} \equiv \frac{a^{2} \dot{\gamma}}{\mathcal{D}} \sim \frac{\left(10^{-8}\right)^{2}}{10^{-10}} \sim 10^{-6}
$$

Since in this work $\mathrm{Pe}<<1$, the dispersions can be considered Brownian.

To determine if the motion of the particles of the coating suspensions investigated in the main article is dictated by that of the carrier fluid, we calculated the Stokes number (St). This is defined as the ratio of the particle relaxation time $\left(\tau_{a}\right)$ to the characteristic flow time scale $\left(\tau_{c}\right)$.

For a concentrated suspension the particle relaxation time is defined as (Jackson, 2000):

$$
\tau_{a} \equiv \frac{(1-\phi)^{2.65}}{1+\left(\phi \rho_{p}\right) /\left[(1-\phi) \rho_{e}\right]} \frac{\rho_{p} a^{3}}{K_{a} \mu}
$$

where $\rho_{s}$ and $\rho_{e}$ are the densities of the particle and carrier fluid, respectively $\left(\sim 10^{3} \mathrm{~kg} / \mathrm{m}^{3}\right), a$ is the particle radius, $\mu$ is the carrier fluid viscosity $\left(\sim 10^{-3} \mathrm{Pas}\right)$ and $K_{a}$ is a coefficient that depends on the shape, size and orientation of the particle (Coussot and Ancey, 1999). The characteristic flow time scale, as discussed in $\mathrm{A} 1$, is the convection characteristic time $\tau_{c}=R / V$. Based on the above, for the values of $\phi$ of interest in our simulations, we get:

$$
\mathrm{St} \equiv \frac{\tau_{a}}{\tau_{c}}=\frac{(1-\phi)^{2.65}}{1+\left(\phi \rho_{p}\right) /\left[(1-\phi) \rho_{e}\right]} \frac{\rho_{p} a^{3}}{K_{a} \mu} \frac{V}{R} \sim 10^{-11}
$$

In the above equation, we have taken $K_{a} \sim a \sim 10^{-8} \mathrm{~m}, V \sim 10^{-3} \mathrm{~m} / \mathrm{s}$ and $R \sim 10^{-2} \mathrm{~m}$ which is true in the simulations performed in the current study.

Since St $<<1$ the motion of the particles in the suspension is mainly dictated by that of the carrier fluid. Therefore, employing the mixture modeling approach is valid for the case studies investigated in this work. 


\section{Acknowledgements}

The authors wish to acknowledge the financial support given to this research project by the Engineering Physical Science Research Council (EPSRC), grant code EP/M507581/1, and Eli Lilly and Company. 


\section{References}

Alleborn, N. and Raszillier, H., 2004. Spreading and sorption of droplets on layered porous substrates. Journal of colloid and interface science, 280(2), pp.449-464.

Batchelor, G.K., 1976. Brownian diffusion of particles with hydrodynamic interaction. Journal of Fluid Mechanics, 74(1), pp.1-29.

Bird, R.B., Stewart, W.E. and Lightfoot, E.N., 2007. Transport phenomena. John Wiley \& Sons.

Bolleddula, D.A., Berchielli, A. and Aliseda, A., 2010. Impact of a heterogeneous liquid droplet on a dry surface: Application to the pharmaceutical industry. Advances in colloid and interface science, 159(2), pp.144-159.

Bradford, S.A., Simunek, J., Bettahar, M., van Genuchten, M.T. and Yates, S.R., 2003. Modeling colloid attachment, straining, and exclusion in saturated porous media. Environmental science \& technology, 37(10), pp.2242-2250.

Bradford, S.A., Yates, S.R., Bettahar, M. and Simunek, J., 2002. Physical factors affecting the transport and fate of colloids in saturated porous media. Water Resources Research, 38(12), pp.63-1.

Brady, J.F., 1993. The rheological behavior of concentrated colloidal dispersions. The Journal of Chemical Physics, 99(1), pp.567-581.

Buyevich, Y.A. and Kapbsov, S.K., 1999. Segregation of a fine suspension in channel flow. Journal of non-newtonian fluid mechanics, 86(1-2), pp.157-184.

Cacuci, D.G., Cacuci, C.G., 2003. Sensitivity and Uncertainty Analysis. Chapman \& Hall/CRC Press, Boca Raton.

Christodoulou, C., Sorensen, E., García-Muñoz, S. and Mazzei, L., 2018. Mathematical modelling of water absorption and evaporation in a pharmaceutical tablet during film coating. Chemical Engineering Science, 175, pp.40-55.

Christodoulou, C., Sorensen, E., García-Muñoz, S. and Mazzei, L., 2020. Mathematical Modeling of Spray Impingement and Film Formation on Pharmaceutical Tablets during Coating. Chemical Engineering Research and Design, 153, pp.768-788.

Civan, F., 2008b. Use exponential functions to correlate temperature dependence. Chemical Engineering Progress, 104(7), pp.46-52.

Civan, F., 2011. Porous media transport phenomena (pp. 152-159). Hoboken: Wiley.

Cole, G.C., 1995. Introduction and overview of pharmaceutical coating. In Pharmaceutical coating technology (pp. 11-15). CRC Press.

Collins, J.H.P., Gladden, L.F., Hardy, I.J. and Mantle, M.D., 2007. Characterizing the evolution of porosity during controlled drug release. Applied Magnetic Resonance, 32(1-2), pp.185-204.

Coussot, P. and Ancey, C., 1999. Rheophysical classification of concentrated suspensions and granular pastes. Physical Review E, 59(4), p.4445.

Craster, R.V. and Matar, O.K., 2009. Dynamics and stability of thin liquid films. Reviews of modern physics, 81(3), p.1131.

Croll, S.G., 1987. Heat and mass transfer in latex paints during drying. JCT, Journal of coatings technology, 59(751), pp.81-92.

De Gennes, P.G., 1985. Wetting: statics and dynamics. Reviews of modern physics, 57(3), p.827.

Delhaye, J.M., 1974. Jump conditions and entropy sources in two-phase systems. Local instant formulation. International Journal of Multiphase Flow, 1(3), pp.395-409. 
Elimelech, M., Gregory, J. and Jia, X., 2013. Particle deposition and aggregation: measurement, modelling and simulation. Butterworth-Heinemann.

Felton, L.A., 2013. Mechanisms of polymeric film formation. International journal of pharmaceutics, 457(2), pp.423-427.

Fluent, A.N.S.Y.S., 2019. Ansys Fluent, Academic Research, Release, 19.

Freireich, B., Kumar, R., Ketterhagen, W., Su, K., Wassgren, C. and Zeitler, J.A., 2015. Comparisons of intra-tablet coating variability using DEM simulations, asymptotic limit models, and experiments. Chemical Engineering Science, 131, pp.197-212.

Hiby, J.W., 1962. Longitudinal and transverse mixing during single-phase flow through granular beds. Interaction between Fluids \& Particles, pp.312-325.

Holloway, W., Aristoff, J.M. and Stone, H.A., 2011. Imbibition of concentrated suspensions in capillaries. Physics of Fluids, 23(8), p.081701.

Iwasaki, T., 1937. Some notes on sand filtration. Journal-American Water Works Association, 29(10), pp.1591-1597.

Jackson, R., 2000. The dynamics of fluidized particles. Cambridge University Press.

Jamshidi, R., Angeli, P. and Mazzei, L., 2019. On the closure problem of the effective stress in the Eulerian-Eulerian and mixture modeling approaches for the simulation of liquid-particle suspensions. Physics of Fluids, 31(1), p.013302.

Ketterhagen, W., Aliseda, A., am Ende, M., Berchielli, A., Doshi, P., Freireich, B. and Prpich, A., 2017. Modeling tablet film-coating processes. In Predictive Modeling of Pharmaceutical Unit Operations (pp. 273-316). Woodhead Publishing.

Krok, A., Vitorino, N., Zhang, J., Frade, J.R. and Wu, C.Y., 2017. Thermal properties of compacted pharmaceutical excipients. International journal of pharmaceutics, 534(1-2), pp.119-127.

Krieger, I.M. and Dougherty, T.J., 1959. A mechanism for non-Newtonian flow in suspensions of rigid spheres. Transactions of the Society of Rheology, 3(1), pp.137-152.

Kutluay, S., Bahadir, A.R. and Özdeş, A., 1997. The numerical solution of one-phase classical Stefan problem. Journal of computational and applied mathematics, 81(1), pp.135-144.

Kiil, S., 2006. Drying of latex films and coatings: Reconsidering the fundamental mechanisms. Progress in Organic Coatings, 57(3), pp.236-250.

Leal, L.G., 2007. Advanced transport phenomena: Fluid mechanics and convective transport processes (Vol. 7). Cambridge University Press.

Léang, M., Pauchard, L., Lee, L.T. and Giorgiutti-Dauphiné, F., 2019. Imbibition on a porous layer: dynamical and mechanical characterization. Soft matter, 15(10), pp.2277-2283.

Möltgen, C.V., Puchert, T., Menezes, J.C., Lochmann, D. and Reich, G., 2012. A novel in-line NIR spectroscopy application for the monitoring of tablet film coating in an industrial scale process. Talanta, 92, pp.26-37.

Mueller, S., Llewellin, E.W. and Mader, H.M., 2009. The rheology of suspensions of solid particles. Proceedings of the Royal Society A: Mathematical, Physical and Engineering Sciences, 466(2116), pp.1201-1228.

Muliadi, A.R. and Sojka, P.E., 2010. A review of pharmaceutical tablet spray coating. Atomization and Sprays, 20(7). 
Niblett, D., Porter, S., Reynolds, G., Morgan, T., Greenamoyer, J., Hach, R., Sido, S., Karan, K. and Gabbott, I., 2017. Development and evaluation of a dimensionless mechanistic pan coating model for the prediction of coated tablet appearance. International journal of pharmaceutics, 528(1-2), pp.180-201.

O'Brien, S.B.G. and Schwartz, L.W., 2002. Theory and modeling of thin film flows. Encyclopedia of surface and colloid science, 1, pp.5283-5297.

Pham, T. and Kumar, S., 2019. Imbibition and evaporation of droplets of colloidal suspensions on permeable substrates. Physical Review Fluids, 4(3), p.034004.

Process Systems Enterprise Ltd., 2019. Release 5.1.1. London, UK. <www.psenterprise.com/gproms>.

Reis Jr, N.C., Griffiths, R.F., Mantle, M.D. and Gladden, L.F., 2003. Investigation of the evaporation of embedded liquid droplets from porous surfaces using magnetic resonance imaging. International Journal of Heat and Mass Transfer, 46(7), pp.1279-1292.

Roy, R.V., Roberts, A.J. and Simpson, M.E., 2002. A lubrication model of coating flows over a curved substrate in space. Journal of Fluid Mechanics, 454, pp.235-261.

Russel, W.B., Russel, W.B., Saville, D.A. and Schowalter, W.R., 1991. Colloidal dispersions. Cambridge university press.

Rutgers, I.R., 1962. Relative viscosity of suspensions of rigid spheres in Newtonian liquids. Rheologica Acta, 2(3), pp.202-210.

Sarkar, A. and Wassgren, C.R., 2009. Simulation of a continuous granular mixer: Effect of operating conditions on flow and mixing. Chemical Engineering Science, 64(11), pp.2672-2682.

Schwartz, L.W. and Weidner, D.E., 1995. Modeling of coating flows on curved surfaces. Journal of engineering mathematics, 29(1), pp.91-103.

Schwartz, L.W. and Eley, R.R., 1998. Simulation of droplet motion on low-energy and heterogeneous surfaces. Journal of Colloid and Interface Science, 202(1), pp.173-188.

Schwartz, L.W., 1999. Theoretical and numerical modeling of coating flow on simple and complex substrates including rheology, drying and Marangoni effects. Advances in coating and drying of thin films, pp.105-128.

Schwartz, L.W., Roy, R.V., Eley, R.R. and Petrash, S., 2001. Dewetting patterns in a drying liquid film. Journal of colloid and interface science, 234(2), pp.363-374.

Siregar, D. P., 2012. Numerical simulation of evaporation and absorption of inkjet printed droplets Eindhoven: Technische Universiteit Eindhoven.

Slattery, J.C., Sagis, L. and Oh, E.S., 2007. Interfacial transport phenomena. Springer Science \& Business Media.

Szeri, A.Z., 2010. Fluid film lubrication. Cambridge university press.

Taylor, J.W. and Winnik, M.A., 2004. Functional latex and thermoset latex films. Jct Research, 1(3), pp.163-190.

Tufenkji, N. and Elimelech, M., 2004. Correlation equation for predicting single-collector efficiency in physicochemical filtration in saturated porous media. Environmental science \& technology, 38(2), pp.529-536.

Weidner, D.E., Schwartz, L.W. and Eley, R.R., 1996. Role of surface tension gradients in correcting coating defects in corners. Journal of colloid and interface science, 179(1), pp.66-75. 
Weidner, D.E., Schwartz, L.W. and Eres, M.H., 1997. Simulation of coating layer evolution and drop formation on horizontal cylinders. Journal of Colloid and interface science, 187(1), pp.243-258.

Yiantsios, S.G. and Higgins, B.G., 2006. Marangoni flows during drying of colloidal films. Physics of Fluids, 18(8), p.082103. 